Working Paper/Document de travail 2014-38

\title{
A New Approach to Infer Changes in the Synchronization of Business Cycle Phases
}

by Danilo Leiva-Leon 
Bank of Canada Working Paper 2014-38

September 2014

\title{
A New Approach to Infer Changes in the Synchronization of Business Cycle Phases
}

\author{
by \\ Danilo Leiva-Leon \\ International Economic Analysis Department \\ Bank of Canada \\ Ottawa, Ontario, Canada K1A OG9 \\ leiv@bankofcanada.ca
}

Bank of Canada working papers are theoretical or empirical works-in-progress on subjects in economics and finance. The views expressed in this paper are those of the author.

No responsibility for them should be attributed to the Bank of Canada. 


\section{Acknowledgements}

I especially thank Maximo Camacho, Marcelle Chauvet, James D. Hamilton and Gabriel Perez-Quiros for their helpful comments and suggestions. I also benefited from conversations with James Morley and Michael T. Owyang. Thanks to the seminar participants at the Bank of Canada, Bank of Mexico, Central Bank of Chile and the University of California, Riverside, and conference participants at the 6th CSDA International Conference on Computational and Financial Econometrics, the 12th Annual Missouri Economics Conference and the XXXVII Symposium of the Spanish Economic Association. A preliminary version of this paper circulated under the name "Monitoring Synchronization of Regional Recessions: A Markov-Switching Network Approach." Supplementary material for this paper can be found on the author's webpage: https://sites.google.com/site/daniloleivaleon/media. 


\begin{abstract}
This paper proposes a Markov-switching framework to endogenously identify the following: (1) regimes where economies synchronously enter recessionary and expansionary phases; and (2) regimes where economies are unsynchronized, essentially following independent business cycles. The reliability of the framework to track changes in synchronization is corroborated with Monte Carlo experiments. An application to the case of U.S. states reports substantial changes over time in the cyclical affiliation patterns of states. Moreover, a network analysis discloses a change in the propagation pattern of aggregate contractionary shocks across states, suggesting that regional economies in the United States have become more interdependent since the early 1990s.
\end{abstract}

JEL classification: E32, C32, C45

Bank classification: Business fluctuations and cycles; Econometric and statistical methods; Regional economic developments

\title{
Résumé
}

Dans cette étude, l'auteur propose un modèle markovien à changement de régime pour repérer de façon endogène : 1 ) les régimes dont les économies entrent simultanément en récession ou dans une phase d'expansion et 2) les régimes dont les économies ne sont pas synchronisées et suivent pour l'essentiel des cycles indépendants. Les simulations de Monte-Carlo viennent confirmer la fiabilité de ce modèle lorsqu'il s'agit de suivre les changements touchant la synchronisation des cycles. Son application au cas des États américains met en évidence des changements importants qui s'opèrent à la longue dans les profils de synchronisation cyclique des États. Par ailleurs, une analyse des réseaux révèle une évolution du profil de propagation de l'ensemble des chocs restrictifs au sein des États, ce qui semble indiquer une interdépendance croissante des économies régionales aux États-Unis depuis le début des années 1990.

Classification JEL : E32, C32, C45

Classification de la Banque : Cycles et fluctuations économiques; Méthodes

économétriques et statistiques; Évolution économique régionale 


\section{Introduction}

The analysis of business cycle synchronization provides crucial information for policymakers in determining the economic regions most sensitive to policy changes or shocks. Most of the related studies have mainly focused on describing the cyclical patterns of economies during a given time span. However, little has been done in terms of assessing potential changes in those patterns, which can be caused by a variety of factors, such as policy modifications, trade agreements, economic unions, aggregate recessionary shocks, etc.

Because of the asymmetric nature of business cycles, multivariate Markov-switching (MS) models (Hamilton (1989)) have become useful tools for analyzing the synchronization of national economies (Smith and Summers (2005) and Camacho and Perez-Quiros (2006)), or regional economies (Owyang et al. (2005) and Hamilton and Owyang (2012)). In these studies, real economic activity is modelled as a function of a latent variable that indicates, at each time period, if the economy is in a recessionary or an expansionary phase. These studies provide an overall picture of the synchronization between the business cycles of different economies, although they are not able to endogenously identify potential synchronization changes. This paper specifically examines how the dependency relationship between the latent variables governing a multivariate MS model changes over time.

The approaches used in the literature to deal with multivariate MS frameworks traditionally assume constant dependency relationships between the latent variables. These can be sorted into two categories. The first category includes studies where the relation is $a$ priori based on the researcher's judgment. Multivariate MS models are usually analyzed under three different settings (Hamilton and Lin (1996) and Anas et al. (2007)). The first refers to the case where all series follow common regime dynamics (Krolzig (1997) and Sims and Zha (2006)). The second, which is the most followed approach, uses totally independent Markov chains (Smith and Summers (2005) and Chauvet and Senyuz (2008)). In the third, the dynamics of one latent variable precedes those of other latent variables (Hamilton and Perez-Quiros (1996) and Cakmakli et al. (2011)), allowing for a possibly different number of lags. ${ }^{1}$ Accordingly, the obtained regime inferences and final interpretations of the model's output may vary substantially depending on the approach chosen. $^{2}$

\footnotetext{
${ }^{1}$ Another type of relationship, under a univariate framework, is presented in Bai and Wang (2011), where the state variable governing the mean of the process is conditional to the one governing the variance of that process.

${ }^{2}$ There is also the case of a general Markovian specification that involves the full transition probability matrix; however, it raises computational difficulties with a large number of series, states or lags. It is
} 
The second category focuses on making a posteriori assessments of the synchronization between MS processes, providing "average" dependency relationship estimates. Work in this line are Guha and Banerji (1998) and Artis et al. (2004), which after estimating different univariate models, compute cross-correlations between the probabilities of being in recession as measures of synchronization. However, as shown in Camacho and PerezQuiros (2006), these approaches may lead to misleading results, since they are biased toward showing relatively low values of synchronization precisely for countries that exhibit synchronized cycles. This suggests that a bivariate framework would provide a better characterization of pairwise synchronization than two univariate models.

Regarding the analysis of pairwise business cycle contemporaneous synchronization, Phillips (1991) points out the two extreme cases presented in the literature: the case of complete independence (two independent Markov processes are hidden in the bivariate specification) and the case of perfect synchronization (only one Markov process for both variables). Similarly, Harding and Pagan (2006) propose a test for the hypothesis that cycles are either unsynchronized or perfectly synchronized. Pesaran and Timmermann (2009) also focus on testing independence between discrete multi-category variables based on canonical correlations. Another similar approach, followed by Camacho and PerezQuiros (2006), Bengoechea et al. (2006), and Leiva-Leon (2014), consists of modelling the data-generating process as a linear combination between the unsynchronized and perfectly synchronized cases. Although these approaches provide inferences on the dependency relationship among the latent variables, they are not able to analyze it in a time-varying fashion.

This paper provides a new approach to infer the time-varying relationship between the latent variables governing multivariate MS models. This information allows us to endogenously identify regimes where two economies enter recessions and expansions synchronously, from regimes where the economies are unsynchronized and experience independent business cycle phases. In contrast to the previous related literature, the proposed filter not only provides a full characterization of the regime inferences, but it also simultaneously provides inferences on the type of synchronicity that both economies experience at each period of time.

The model is estimated by Gibbs sampling and its reliability is assessed with Monte Carlo experiments, which identify it as a suitable approach to track changes in the synchronization of cycles. Moreover, the obtained pairwise synchronizations can be easily converted into measures of dissimilarity, which can be interpreted as cyclical distances and used in assessing changes in the clustering and interdependence patterns that could be experienced by not only two, but many, economies. This is done by relying on network

also less straightforward to interpret, and does not allow us to endogenously infer the type of relationship between the latent variables. 
analysis, where economies take the interpretation of nodes, with links between pairs of nodes given by the estimated synchronicity, fully characterizing a business cycle network governed by Markovian dynamics.

The proposed framework is applied to investigate potential variations in the cyclical interdependence between U.S. states, obtaining three main findings. First, the results report the existence of interdependence cycles, which are associated with recessions as identified by the National Bureau of Economic Research (NBER). Such cycles are defined as periods characterized by low cyclical heterogeneity across states, experienced during the recessionary and recovery phases, followed by longer periods of high cyclical heterogeneity, which occurs during the phases of stable growth. Second, there are substantial variations in the grouping pattern of states over time, going from a scheme characterized by several clusters of states to a core and periphery structure, composed of highly and lowly synchronized states, respectively. Third, the network analysis documents a change in the propagation pattern of contractionary shocks across states. Until the 1990s, recessions were characterized by the spread of shocks mainly across a few big states in terms of their share of GDP. Since that time, recessionary shocks have been more uniformly spread across all states, suggesting that regions of the U.S. economy have become more interdependent over the past two decades.

The paper is structured as follows. Section 2 presents the proposed time-varying synchronization approach, describes the filtering algorithm and reports the Monte Carlo simulation results. Section 3 analyzes the dynamic synchronization of business cycle phases in U.S. states, relying on bivariate, multivariate and network analyses. Finally, Section 4 concludes.

\section{The Model}

Let $y_{i, t}$ be the growth rate of an economic activity index of economy $i$, which can be modelled as a function of a latent or unobserved state variable $\left(S_{i, t}\right)$ that indicates whether the economy is in a recessionary or expansionary regime, an idiosyncratic component, $\epsilon_{i, t}$, and a set of additional parameters, $\theta_{i}$. Accordingly, for $i=a, b$,

$$
\begin{aligned}
& y_{a, t}=f\left(S_{a, t}, \epsilon_{a, t}, \theta_{a}\right) \\
& y_{b, t}=f\left(S_{b, t}, \epsilon_{b, t}, \theta_{b}\right) .
\end{aligned}
$$

The goal of this section is to provide assessments on the synchronization between $S_{a, t}$ and $S_{b, t}$ for each period of time; that is,

$$
\operatorname{sync}\left(S_{a, t}, S_{b, t}\right)=\operatorname{Pr}\left(S_{a, t}=S_{b, t}\right), \text { for } t=1, \ldots, T \text {. }
$$


Following Owyang et al. (2005) and Hamilton and Owyang (2012), who rely on AR(0) MS specification, the following tractable bivariate two-state Markov-switching specification is considered:

$$
\left[\begin{array}{l}
y_{a, t} \\
y_{b, t}
\end{array}\right]=\left[\begin{array}{c}
\mu_{a, 0}+\mu_{a, 1} S_{a, t} \\
\mu_{b, 0}+\mu_{b, 1} S_{b, t}
\end{array}\right]+\left[\begin{array}{c}
\varepsilon_{a, t} \\
\varepsilon_{b, t}
\end{array}\right],\left[\begin{array}{l}
\varepsilon_{a, t} \\
\varepsilon_{b, t}
\end{array}\right] \sim N\left(\left[\begin{array}{l}
0 \\
0
\end{array}\right],\left[\begin{array}{cc}
\sigma_{a}^{2} & \sigma_{a b} \\
\sigma_{a b} & \sigma_{b}^{2}
\end{array}\right]\right) .
$$

It is worth noting that the results derived in this section can be straightforwardly extended to specifications including lags in the dynamics. However, Camacho and PerezQuiros (2007) show that positive autocorrelation in macroeconomic time series can be better captured by shifts between business cycle states rather than by the standard autoregressive coefficients. The model can also be extended to allow for regime switching in the variance-covariance matrix; however, since the empirical application focuses on the period after the Great Moderation, such a feature is not included in the model.

When $S_{k, t}=0$, the state variable $S_{k, t}$ indicates that $y_{k t}$ is in regime 0 with a mean equal to $\mu_{k, 0}$. When $S_{k, t}=1, y_{k t}$ is in regime 1 with a mean equal to $\mu_{k, 0}+\mu_{k, 1}$, for $k=a, b$. Moreover, $S_{a, t}$ and $S_{b, t}$ evolve according to irreducible two-state Markov chains, whose transition probabilities are given by

$$
\operatorname{Pr}\left(S_{k, t}=j \mid S_{k, t-1}=i\right)=p_{k, i j}, \text { for } i, j=0,1 \text { and } k=a, b .
$$

To characterize the dynamics of $y_{t}=\left[y_{a, t}, y_{b, t}\right]^{\prime}$, the information contained in $S_{a, t}$ and $S_{b, t}$ can be summarized in the state variable, $S_{a b, t}$, which accounts for the possible combinations that the vector $\mu_{S_{a b, t}}=\left[\mu_{a, 0}+\mu_{a, 1} S_{a, t}, \mu_{b, 0}+\mu_{b, 1} S_{b, t}\right]^{\prime}$ could take through the different regimes:

$$
S_{a b, t}=\left\{\begin{array}{l}
1, \text { if } S_{a, t}=0, S_{b, t}=0 \\
2, \text { if } S_{a, t}=0, S_{b, t}=1 \\
3, \text { if } S_{a, t}=1, S_{b, t}=0 \\
4, \text { if } S_{a, t}=1, S_{b, t}=1
\end{array} .\right.
$$

Similar to Harding and Pagan (2006), the objective of the proposed model is to differentiate regimes where the phases of $y_{a, t}$ and $y_{b, t}$ are unsynchronized, implying that $S_{a, t}$ and $S_{b, t}$ follow independent dynamics; that is,

$$
\operatorname{Pr}\left(S_{a, t}=j_{a}, S_{b, t}=j_{b}\right)=\operatorname{Pr}\left(S_{a, t}=j_{a}\right) \operatorname{Pr}\left(S_{b, t}=j_{b}\right),
$$

from regimes where the phases of $y_{a, t}$ and $y_{b, t}$ are fully synchronized, entering expansions and recessions synchronously, implying that $S_{a, t}=S_{b, t}=S_{t}$; that is,

$$
\operatorname{Pr}\left(S_{a, t}=j_{a}, S_{b, t}=j_{b}\right)=\operatorname{Pr}\left(S_{t}=j\right) .
$$


In order to do so, I introduce into the framework another latent variable, $V_{t}$, that takes the value of 1 if business cycle phases are in a synchronized regime, and the value of 0 if they are under an unsynchronized regime at time $t$; that is,

$$
V_{t}=\left\{\begin{array}{c}
0 \text { if } S_{a, t} \text { and } S_{b, t} \text { are unsynchronized } \\
1 \text { if } S_{a, t} \text { and } S_{b, t} \text { are synchronized }
\end{array} .\right.
$$

The latent variable $V_{t}$ also evolves according to an irreducible two-state Markov chain whose transition probabilities are given by

$$
\operatorname{Pr}\left(V_{t}=j_{v} \mid V_{t-1}=i_{v}\right)=p_{v, k l}, \quad \text { for } i_{v}, j_{v}=0,1 \text {. }
$$

The advantage of introducing $V_{t}$, rather than analyzing the general Markovian specification with the full transition probability matrix, as in Sims et al. (2008), is that all the information about the dependency relationship between the latent variables remains summarized in a single variable, $V_{t}$, providing an easy-to-interpret way of assessing synchronization changes. It is also able to provide information about the expected duration of regimes where economies are synchronized or unsynchronized based on their associated transition probabilities. Notice that the analysis in this paper focuses on dependency, not on correlations, since the objective is to determine if two economies are either synchronized or unsynchronized.

Accordingly, there is an enlargement of the set of regimes in Equation (6), which remains fully characterized by the latent variable $S_{a b, t}^{*}$, that simultaneously collects information regarding joint dynamics, individual dynamics and their dependency relationship over time:

$$
S_{a b, t}^{*}=\left\{\begin{array}{l}
1, \text { if } S_{a, t}=0, S_{b, t}=0, V_{t}=0 \\
2, \text { if } S_{a, t}=0, S_{b, t}=1, V_{t}=0 \\
3, \text { if } S_{a, t}=1, S_{b, t}=0, V_{t}=0 \\
4, \text { if } S_{a, t}=1, S_{b, t}=1, V_{t}=0 \\
5, \text { if } S_{a, t}=0, S_{b, t}=0, V_{t}=1 \\
6, \text { if } S_{a, t}=0, S_{b, t}=1, V_{t}=1 \\
7, \text { if } S_{a, t}=1, S_{b, t}=0, V_{t}=1 \\
8, \text { if } S_{a, t}=1, S_{b, t}=1, V_{t}=1
\end{array} .\right.
$$

Inferences on the latent variable $S_{a b, t}^{*}$, can be computed by conditioning on $V_{t}^{3}$ :

$$
\begin{aligned}
\operatorname{Pr}\left(S_{a b, t}^{*}\right. & \left.=j_{a b}^{*}\right)=\operatorname{Pr}\left(S_{a, t}=j_{a}, S_{b, t}=j_{b}, V_{t}=j_{v}\right) \\
& =\operatorname{Pr}\left(S_{a, t}=j_{a}, S_{b, t}=j_{b} \mid V_{t}=j_{v}\right) \operatorname{Pr}\left(V_{t}=j_{v}\right)
\end{aligned}
$$

\footnotetext{
${ }^{3}$ Notice that states 6 and 7 in Equation (11) are truncated to zero by construction, since the two state variables cannot be in different states if they are perfectly synchronized, i.e., $\operatorname{Pr}\left(S_{a, t}=j_{a}, S_{b, t}=j_{b} \mid V_{t}=\right.$ 1) $=0$ for any $j_{a} \neq j_{b}$.
} 
where $\operatorname{Pr}\left(S_{a, t}=j_{a}, S_{b, t}=j_{b} \mid V_{t}=j_{v}\right)$ indicates the inferences on the dynamics of $S_{a b, t}$, conditional on total independence if $V_{t}=0$, or conditional on full dependence if $V_{t}=1$. In the former case, the joint probability of $S_{a b, t}^{*}$ is given by

$$
\begin{aligned}
\operatorname{Pr}\left(S_{a, t}\right. & \left.=j_{a}, S_{b, t}=j_{b}, V_{t}=0\right)=\operatorname{Pr}\left(S_{a, t}=j_{a}, S_{b, t}=j_{b} \mid V_{t}=0\right) \operatorname{Pr}\left(V_{t}=0\right) \\
& =\operatorname{Pr}\left(S_{a, t}=j_{a}\right) \operatorname{Pr}\left(S_{b, t}=j_{b}\right) \operatorname{Pr}\left(V_{t}=0\right)
\end{aligned}
$$

while, in the latter case, it is given by

$$
\begin{aligned}
\operatorname{Pr}\left(S_{a, t}\right. & \left.=j_{a}, S_{b, t}=j_{b}, V_{t}=1\right)=\operatorname{Pr}\left(S_{a, t}=j_{a}, S_{b, t}=j_{b} \mid V_{t}=1\right) \operatorname{Pr}\left(V_{t}=1\right) \\
& =\operatorname{Pr}\left(S_{t}=j\right) \operatorname{Pr}\left(V_{t}=1\right) .
\end{aligned}
$$

Therefore, inferences on the state variable $S_{a b, t}$, in Equation (6), after accounting for synchronization, can be easily recovered by integrating $\operatorname{Pr}\left(S_{a, t}=j_{a}, S_{b, t}=j_{b}, V_{t}=j_{v}\right)$ through $V_{t}$; that is,

$$
\begin{aligned}
\operatorname{Pr}\left(S_{a, t}\right. & \left.=j_{a}, S_{b, t}=j_{b}\right)=\operatorname{Pr}\left(V_{t}=1\right) \operatorname{Pr}\left(S_{t}=j\right)+ \\
\left(1-\operatorname{Pr}\left(V_{t}\right.\right. & =1)) \operatorname{Pr}\left(S_{a, t}=j_{a}\right) \operatorname{Pr}\left(S_{b, t}=j_{b}\right),
\end{aligned}
$$

which implies that the joint dynamics of $S_{a, t}$ and $S_{b, t}$ remain characterized by a weighted average between the extreme dependent and independent cases, where the weights assigned to each of them are endogenously determined by

$$
\operatorname{Pr}\left(V_{t}=1\right)=\delta_{t}^{a b}
$$

Therefore, from now on, the term $\delta_{t}^{a b}$ will be referred to as the dynamic synchronicity between $S_{a, t}$ and $S_{b, t}$.

\subsection{Filtering Algorithm}

This section develops an extension of the Hamilton (1994) algorithm to estimate the model described in Equations (4) and (15). The algorithm is composed of two unified steps. In the first one, the goal is the computation of the likelihoods, while in the second, the goal is the prediction and updating of probabilities.

STEP 1: The parameters of the model are assumed to be known for the moment and are collected in the vector

$$
\theta=\left(\mu_{a, 0}, \mu_{a, 1}, \mu_{b, 0}, \mu_{b, 1}, \sigma_{a}^{2}, \sigma_{b}^{2}, \sigma_{a b}, p_{a, 00}, p_{a, 11}, p_{b, 00}, p_{b, 11}, p_{00}, p_{11}, p_{v, 00}, p_{v, 11}\right)^{\prime}
$$

The conditional joint density corresponding to the state variable that fully characterizes 
the model's dynamics, $S_{a b, t}^{*}$, can be expressed as a function of its components,

$$
f\left(y_{t}, S_{a b, t}^{*}=j_{a b}^{*} \mid \psi_{t-1} ; \theta\right)=f\left(y_{t}, S_{a, t}=j_{a}, S_{b, t}=j_{b}, V_{t}=j_{v} \mid \psi_{t-1} ; \theta\right)
$$

which is the product of the density, conditional on the realization of the set of regimes times the probability of occurrence of such realizations,

$$
\begin{aligned}
f\left(y_{t}, S_{a, t}\right. & \left.=j_{a}, S_{b, t}=j_{b}, V_{t}=j_{v} \mid \psi_{t-1} ; \theta\right)=f\left(y_{t} \mid S_{a, t}=j_{a}, S_{b, t}=j_{b}, V_{t}=j_{v}, \psi_{t-1} ; \theta\right) \times \\
\operatorname{Pr}\left(S_{a, t}\right. & \left.=j_{a}, S_{b, t}=j_{b}, V_{t}=j_{v} \mid \psi_{t-1} ; \theta\right) .
\end{aligned}
$$

The trivariate probability of $S_{a, t}=j_{a}, S_{b, t}=j_{b}$ and $V_{t}=j_{v}$ is obtained by using conditional probabilities,

$$
\begin{aligned}
\operatorname{Pr}\left(S_{a, t}\right. & \left.=j_{a}, S_{b, t}=j_{b}, V_{t}=j_{v} \mid \psi_{t-1} ; \theta\right)=\operatorname{Pr}\left(S_{a, t}=j_{a}, S_{b, t}=j_{b} \mid V_{t}=j_{v}, \psi_{t-1} ; \theta\right) \times \\
\operatorname{Pr}\left(V_{t}\right. & \left.=j_{v} \mid \psi_{t-1} ; \theta\right),
\end{aligned}
$$

where the term $\operatorname{Pr}\left(S_{a, t}=j_{a}, S_{b, t}=j_{b} \mid V_{t}=j_{v}, \psi_{t-1} ; \theta\right)$ is fully characterized with the results derived in Equations (13) and (14). Thus, Equation (20) remains a function of only $\operatorname{Pr}\left(S_{k, t}=j_{k} \mid \psi_{t-1} ; \theta\right)$ for $k=a, b, \operatorname{Pr}\left(V_{t}=j_{v} \mid \psi_{t-1} ; \theta\right)$ and $\operatorname{Pr}\left(S_{t}=j \mid \psi_{t-1} ; \theta\right)$. The steady state or ergodic probabilities can be used as starting values to initialize the filter.

In order to make inferences on the evolution of single-state variables, the marginal densities are obtained as

$$
\begin{aligned}
& f\left(y_{t}, S_{a, t}\right.\left.=j_{a} \mid \psi_{t-1} ; \theta\right)=\sum_{j_{b}=0}^{1} \sum_{j_{v}=0}^{1} f\left(y_{t}, S_{a, t}=j_{a}, S_{b, t}=j_{b}, V_{t}=j_{v} \mid \psi_{t-1} ; \theta\right), \\
& f\left(y_{t}, S_{b, t}=j_{b} \mid \psi_{t-1} ; \theta\right)=\sum_{j_{a}=0}^{1} \sum_{j_{v}=0}^{1} f\left(y_{t}, S_{a, t}=j_{a}, S_{b, t}=j_{b}, V_{t}=j_{v} \mid \psi_{t-1} ; \theta\right), \\
& f\left(y_{t}, V_{t}=j_{v} \mid \psi_{t-1} ; \theta\right)=\sum_{j_{a}=0}^{1} \sum_{j_{b}=0}^{1} f\left(y_{t}, S_{a, t}=j_{a}, S_{b, t}=j_{b}, V_{t}=j_{v} \mid \psi_{t-1} ; \theta\right) .
\end{aligned}
$$

The marginal density associated the state variable $S_{t}$ requires a special treatment. When it is assumed that the model's dynamics are governed by only one state variable, i.e., $S_{a, t}=S_{b, t}=S_{t}$, the density in Equation (18) collapses to $f^{\dagger}\left(y_{t}, S_{t}=j \mid \psi_{t-1} ; \theta\right)$, where

$$
\begin{aligned}
& f^{\dagger}\left(y_{t}, S_{t}=0 \mid \psi_{t-1} ; \theta\right)=f\left(y_{t}, S_{a, t}=0, S_{b, t}=0, V_{t}=1 \mid \psi_{t-1} ; \theta\right) \\
& f^{\dagger}\left(y_{t}, S_{t}=1 \mid \psi_{t-1} ; \theta\right)=f\left(y_{t}, S_{a, t}=1, S_{b, t}=1, V_{t}=1 \mid \psi_{t-1} ; \theta\right)
\end{aligned}
$$


Accordingly, the density of $y_{t}$, conditional on the past observables, is given by

$$
f\left(y_{t} \mid \psi_{t-1} ; \theta\right)=\sum_{j_{a}=0}^{1} \sum_{j_{b}=0}^{1} \sum_{j_{v}=0}^{1} f\left(y_{t}, S_{a, t}=j_{a}, S_{b, t}=j_{b}, V_{t}=j_{v} \mid \psi_{t-1} ; \theta\right),
$$

and under the assumption that $S_{a, t}=S_{b, t}=S_{t}$, it is given by

$$
f^{\dagger}\left(y_{t} \mid \psi_{t-1} ; \theta\right)=\sum_{j=0}^{1} f^{\dagger}\left(y_{t}, S_{t}=j \mid \psi_{t-1} ; \theta\right)
$$

STEP 2: Once $y_{t}$ is observed at the end of time $t$, the prediction probabilities $\operatorname{Pr}\left(S_{k, t}=\right.$ $\left.j_{k} \mid \psi_{t-1} ; \theta\right)$ for $k=a, b, \operatorname{Pr}\left(V_{t}=j_{v} \mid \psi_{t-1} ; \theta\right)$ and $\operatorname{Pr}\left(S_{t}=j \mid \psi_{t-1} ; \theta\right)$ can be updated:

$$
\begin{aligned}
\operatorname{Pr}\left(S_{a, t}\right. & \left.=j_{a} \mid \psi_{t} ; \theta\right)=\frac{f\left(y_{t}, S_{a, t}=j_{a} \mid \psi_{t-1} ; \theta\right)}{f\left(y_{t} \mid \psi_{t-1} ; \theta\right)} \\
\operatorname{Pr}\left(S_{b, t}\right. & \left.=j_{b} \mid \psi_{t} ; \theta\right)=\frac{f\left(y_{t}, S_{b, t}=j_{b} \mid \psi_{t-1} ; \theta\right)}{f\left(y_{t} \mid \psi_{t-1} ; \theta\right)} \\
\operatorname{Pr}\left(V_{t}\right. & \left.=l \mid \psi_{t} ; \theta\right)=\frac{f\left(y_{t}, V_{t}=l \mid \psi_{t-1} ; \theta\right)}{f\left(y_{t} \mid \psi_{t-1} ; \theta\right)} \\
\operatorname{Pr}\left(S_{t}\right. & \left.=j \mid \psi_{t} ; \theta\right)=\frac{f^{\dagger}\left(y_{t}, S_{t}=j \mid \psi_{t-1} ; \theta\right)}{f^{\dagger}\left(y_{t} \mid \psi_{t-1} ; \theta\right)}
\end{aligned}
$$

Forecasts of the updated probabilities in Equations (28) to (31) are done by using the corresponding transition probabilities $p_{a, i j}, p_{b, i j}, p_{i j}, p_{v, i j}$, in the vector $\theta$, for $S_{a, t}, S_{b, t}, S_{t}$ and $V_{t}$, respectively:

$$
\begin{aligned}
\operatorname{Pr}\left(S_{k, t+1}\right. & \left.=j_{k} \mid \psi_{t} ; \theta\right)=\sum_{i_{k}=0}^{1} \operatorname{Pr}\left(S_{k, t+1}=j_{k}, S_{k, t}=i_{k} \mid \psi_{t} ; \theta\right) \\
& =\sum_{i_{k}=0}^{1} \operatorname{Pr}\left(S_{k, t+1}=j_{k} \mid S_{k, t}=i_{k}\right) \operatorname{Pr}\left(S_{k, t}=i_{k} \mid \psi_{t} ; \theta\right), \text { for } k=a, b \\
\operatorname{Pr}\left(V_{t+1}\right. & \left.=j_{v} \mid \psi_{t} ; \theta\right)=\sum_{i=0}^{1} \operatorname{Pr}\left(V_{t+1}=j_{v}, V_{t}=i_{v} \mid \psi_{t} ; \theta\right) \\
& =\sum_{i=0}^{1} \operatorname{Pr}\left(V_{t+1}=j_{v} \mid V_{t}=i_{v}\right) \operatorname{Pr}\left(V_{t}=i_{v} \mid \psi_{t} ; \theta\right) \\
\operatorname{Pr}\left(S_{t+1}\right. & \left.=j \mid \psi_{t} ; \theta\right)=\sum_{i=0}^{1} \operatorname{Pr}\left(S_{t+1}=j, S_{t}=i \mid \psi_{t} ; \theta\right) \\
& =\sum_{i=0}^{1} \operatorname{Pr}\left(S_{t+1}=j \mid S_{t}=i\right) \operatorname{Pr}\left(S_{t}=i \mid \psi_{t} ; \theta\right)
\end{aligned}
$$

Finally, the above forecasted probabilities are used to predict inferences on the real- 
izations of $S_{a b, t+1}^{*}$, relying on Equation (20):

$$
\begin{aligned}
\operatorname{Pr}\left(S_{a, t+1}\right. & \left.=j_{a}, S_{b, t+1}=j_{b}, V_{t+1}=j_{v} \mid \psi_{t} ; \theta\right)=\operatorname{Pr}\left(S_{a, t+1}=j_{a}, S_{b, t+1}=j_{b} \mid V_{t+1}=j_{v}, \psi_{t} ; \theta\right) \times \\
\operatorname{Pr}\left(V_{t+1}\right. & \left.=j_{v} \mid \psi_{t} ; \theta\right),
\end{aligned}
$$

where Equation (35) remains a function of $\operatorname{Pr}\left(S_{k, t+1}=j_{k} \mid \psi_{t} ; \theta\right)$ for $k=a, b, \operatorname{Pr}\left(V_{t+1}=\right.$ $\left.j_{v} \mid \psi_{t} ; \theta\right)$ and $\operatorname{Pr}\left(S_{t+1}=j \mid \psi_{t} ; \theta\right)$.

By iterating these two steps for $t=1,2, \ldots, T$, the algorithm provides simultaneous inferences on $S_{a, t}, S_{b, t}$ and their dynamic synchronicity $\delta_{t}^{a b}$, defined in Equation (16).

Regarding the estimation of the parameters, notice that, as the number of possible states increases, the likelihood function could be characterized by several local maximums, causing strong convergence problems in performing maximum likelihood estimations, as shown in Boldin (1996). Hence, given the high number of combinations of states through which the likelihood is conditioned in Equation (26), the set of parameters $\theta$ along with the inferences on the state variables are estimated by using Bayesian methods. Specifically, a multivariate version of the approach in Kim and Nelson (1999), which applies Gibbs sampling procedures, is used. The estimation method is explained in detail in the Appendix.

\subsection{Simulation Study}

In order to validate the reliability of the proposed approach to assess changes in the synchronization of business cycle phases, I rely on the use of Monte Carlo experiments. Each simulation consists of two steps. First, the generation of two stochastic processes subject to regime switching that experience one or more synchronization changes. Second, by letting the econometrician observe only the generated data, but not the data-generating process, the proposed filter in Section 2.1 along with the Gibbs sampler, are applied to obtain estimates of the model's parameters, probabilities of recession for each economy, and, more importantly, the inferences on synchronization changes. I then address how well the parameter estimates and inferences match the real ones. ${ }^{4}$

Given a sample of size $T$, the data-generating process consists of generating a first-order Markovian process, $S_{a, t}$, with a transition probability matrix,

$$
P_{a}^{*}=\left(\begin{array}{cc}
p_{a, 00}^{*} & 1-p_{a, 11}^{*} \\
1-p_{a, 00}^{*} & p_{a, 11}^{*}
\end{array}\right)
$$

and an error term, $e_{a, t}^{I}$, drawn from an $N(0,1)$. Then, given a vector of means $\left[\mu_{a, 0}^{*}, \mu_{a, 1}^{*}\right]^{\prime}$

\footnotetext{
${ }^{4}$ It is important to note that the filter's performance is assessed by simulations, under the assumption that the model is correctly specified. An interesting extension in this line of research could be assessing such performance by relaxing this assumption.
} 
and standard error $\sigma_{a}^{*}$, I generate a process $y_{a, t}^{I}$ as follows:

$$
y_{a, t}^{I}=\mu_{a, 0}^{*}+\mu_{a, 1}^{*} S_{a, t}+\sigma_{a}^{*} e_{a, t}^{I},
$$

and given a vector of means $\left[\mu_{b, 0}^{*}, \mu_{b, 1}^{*}\right]^{\prime}$, standard error $\sigma_{b}^{*}$ and transition probabilities $p_{b, 00}^{*}$ and $p_{b, 11}^{*}$, the same procedure is repeated to independently generate

$$
y_{b, t}^{I}=\mu_{b, 0}^{*}+\mu_{b, 1}^{*} S_{b, t}+\sigma_{b}^{*} e_{b, t}^{I}
$$

where $S_{b, t}$ is a first-order Markovian process and $e_{b, t}^{I}$ is drawn from an $N(0,1)$. Next, another Markovian process, $S_{t}$, is generated by using the transition matrix

$$
P_{a b}^{*}=\left(\begin{array}{cc}
p_{00}^{*} & 1-p_{11}^{*} \\
1-p_{00}^{*} & p_{11}^{*}
\end{array}\right)
$$

and an error-term vector $\left[e_{a, t}^{D}, e_{b, t}^{D}\right]^{\prime}$ is drawn from a bivariate normal distribution. Then, given the two vectors of means $\left[\mu_{a, 0}^{*}, \mu_{a, 1}^{*}\right]^{\prime},\left[\mu_{b, 0}^{*}, \mu_{b, 1}^{*}\right]^{\prime}$, standard errors $\sigma_{a}^{*}, \sigma_{b}^{*}$, and a parameter $\sigma_{a b}^{*}$, I generate

$$
\left[\begin{array}{c}
y_{a, t}^{D} \\
y_{b, t}^{D}
\end{array}\right]=\left[\begin{array}{c}
\mu_{a, 0}^{*}+\mu_{a, 1}^{*} S_{t} \\
\mu_{b, 0}^{*}+\mu_{b, 1}^{*} S_{t}
\end{array}\right]+\left[\begin{array}{cc}
\sigma_{a}^{*} & \sigma_{a b}^{*} \\
\sigma_{a b}^{*} & \sigma_{b}^{*}
\end{array}\right]\left[\begin{array}{c}
e_{a, t}^{D} \\
e_{b, t}^{D}
\end{array}\right]
$$

The information generated so far can be collected in two vectors, one in which two stochastic processes are driven by two Markov-switching variables independent from each other, $y_{t}^{I}=\left[y_{a, t}^{I}, y_{b, t}^{I}\right]^{\prime}$, and the other where two stochastic processes are governed by only one Markov-switching dynamic, $y_{t}^{D}=\left[y_{a, t}^{D}, y_{b, t}^{D}\right]^{\prime}$.

The premise of this paper is that, during some regimes, the output growth of two economies can follow dynamics similar to those in $y_{t}^{D}$, while during other regimes, things can change in one, or both, of the economies, leading their joint dynamics to behave in the same way as those in $y_{t}^{I}$, following independent patterns. To mimic this situation, I start analyzing the simplest case in which there is just one synchronization change in a sample of size $T$, occurring at time $\tau$, with $1<\tau<T .{ }^{5}$ Then, I let $y_{t}=\left[y_{a, t}, y_{b, t}\right]^{\prime}$ be the observed output growth of two economies, which comes from the following unobserved data-generating process:

$$
y_{t}=\left\{\begin{array}{c}
y_{t}^{D}, \text { for } t=1, \ldots, \tau \\
y_{t}^{I}, \text { for } t=\tau+1, \ldots, T
\end{array},\right.
$$

\footnotetext{
${ }^{5}$ The selection of $\tau$ is based on a random draw $u$, generated from a uniform distribution $U[0,1]$, i.e., $\hat{\tau}=u T$, then $\hat{\tau}$ is rounded to the nearest integer number to obtain $\tau$. Also, the use of draws of $\tau$ equal to the boundaries, i.e., 1 or $T$, is avoided.
} 
which can be alternatively expressed as

$$
y_{t}=y_{t}^{D} V_{t}+\left(1-V_{t}\right) y_{t}^{I},
$$

where $V_{t}$ is an indicator variable of synchronization, whose dynamics are described by

$$
\left\{V_{t}\right\}_{1}^{T}=\left[\begin{array}{c}
\mathbf{1}_{\tau} \\
\mathbf{0}_{T-\tau}
\end{array}\right]
$$

with $\mathbf{1}_{\tau}$ being a vector, with entries equal to one, of size $\tau$, and $\mathbf{0}_{T-\tau}$ a zero vector of size $T-\tau$. The case of one synchronization change can be easily extended to mimic the case of $Z$ synchronization changes, occurred at $\tau_{1}, \tau_{2}, \ldots, \tau_{Z}$, with $1<\tau_{1}<\tau_{2}<\ldots<\tau_{Z}<T$, just by appropriately modifying the dynamics in $\left\{V_{t}\right\}_{1}^{T}$.

Since the data-generating process and parameters are unknown by the econometrician, the Gibbs sampler is used to estimate the model's parameters, the probabilities of recession for each economy and, more importantly, inferences on the dynamics of $V_{t}$, by relying on the filtering algorithm proposed in Section 2.1. The criterion used to assess the performance of the regime inferences and the synchronization is the Quadratic Probability Score (QPS), defined as

$$
\operatorname{QPS}(\Xi)=\frac{1}{T} \sum_{t=1}^{T}\left(\Xi-\operatorname{Pr}\left(\Xi=1 \mid \psi_{T}\right)\right)^{2}, \text { for } \Xi=S_{a, t}, S_{b, t}, V_{t} .
$$

To illustrate the filtering and estimation strategy's performance, Figure 1 plots one simulation for the cases in which there is one, two and three synchronization changes in a sample of 200 periods, i.e., for $z=1,2,3$, with $T=200$. For each case, the top charts plot the two observed time series, $y_{a, t}$ and $y_{b, t}$, generated with the parameter values in Table 1 and by using Equation (42), along with the unobserved dynamics of $V_{t}$. Both time series show strong coherence in phases when $V_{t}=1$, and the opposite occurs when $V_{t}=0$. The two middle charts plot the probabilities of recession associated with each time series, i.e., $\operatorname{Pr}\left(S_{k, t}=0 \mid \psi_{T}\right)$, for $k=a, b$, showing values near to one when the corresponding time series reports consecutive negative values; the dynamics of $V_{t}$ is also plotted as reference. Finally, the bottom charts plot the computed inferences on the synchronization changes, i.e., $\operatorname{Pr}\left(V_{t}=1\right)$, along with the true dynamics of $V_{t}$, showing their close relation in all three cases and providing insight into the satisfactory performance of the proposed framework for assessing synchronization changes.

This experiment is replicated $M=1,000$ times for $Z=6$ different cases. Each case corresponds to $z$ changes in synchronization, for $z=1,2,3,4,5$, and the last case considers a random number of synchronization changes, i.e., unlike predefining the dynamics of $V_{t}$ as in Equation (43), it is modelled as a first-order Markov chain with transition probabilities 
$p_{V, 00}^{*}$ and $p_{V, 11}^{*}$, i.e. $z=f\left(V_{t}\right){ }^{6}$

The result of the Monte Carlo simulations are reported in Table 2, showing the average over the $M$ replications of each estimated parameter

$$
\theta_{z}^{*}=\frac{1}{M} \sum_{m=1}^{M} \theta_{z}^{*(m)}
$$

where $\theta_{z}^{*(m)}$ corresponds to the vector of parameters, as defined in Equation (17), associated to the $m$ th replica and the $z$ th case. All parameter estimates appear to be unbiased for the different values of $z$. However, two features deserve attention. First, the stochastic process with the highest difference of the within-regime means, in this case $y_{b, t}$, shows more accurate estimates, meaning that higher differences provide a better identification of the phases of the business cycles. ${ }^{7}$ Second, the accuracy in the estimation of the transition probabilities decreases when $z=f\left(V_{t}\right)$, owing to the high number of synchronization changes and the short duration of each change generated by letting $V_{t}$ follow Markovian dynamics.

Regarding the performance of the regime inferences, Table 3 reports the averages over the $M$ replications with the QPS associated with the state variables $S_{a, t}, S_{b, t}$ and $V_{t}$, which can be interpreted as the average over the $M$ replications of the squared deviation from the generated business cycles:

$$
Q P S(\Xi)_{z}=\frac{1}{M} \sum_{m=1}^{M} Q P S(\Xi)_{z}^{(m)}, \text { for } \Xi=S_{a, t}, S_{b, t}, V_{t}
$$

where $Q P S(\Xi)_{z}^{(m)}$, as defined in Equation (44), corresponds to the $m$ th replica and the $z$ th case. The results indicate that, although inferences on the state variables in general present high precision, the ones associated with the time series with the highest difference of the within-regime means, $y_{b, t}$, are the most accurate. The main message of the table is that the precision of the inferences decreases as the number of synchronization changes, $k$, increases. This feature can also be observed by looking at the histograms of the $M$ replications plotted in Figure 2, in particular, the ones associated with $Q P S\left(V_{t}\right)$. However, it is natural to think of synchronization changes as events that do not occur as often as the business cycle phases of an economy. They may require longer periods of time to take place, since they originate from changes in the structural relationships among economies. This suggests that the proposed model is suitable for accurately inferring synchronization changes of business cycle phases.

\footnotetext{
${ }^{6}$ This is done using the corresponding values given in Table 1.

${ }^{7}$ The parameters associated with the variance-covariance matrix of $y_{t}$ are not analyzed in Table 2 because the variance-covariance matrix changes through the regimes of dependence and are therefore not comparable with the estimated ones.
} 


\section{Monitoring U.S. States Business Cycles Synchronization}

The most recent global financial crisis has stimulated interest in the study of the sources and propagation of contractionary episodes, calling for a more careful look at the disaggregation of business cycles in order to assess the mechanisms underlying economic fluctuations.

On the one hand, recent work by Acemoglu et al. (2012), which relies on network analysis, finds that sectoral interconnections capture the possibility of "cascade effects," whereby productivity shocks to a sector propagate not only to its immediate downstream customers, but also to the rest of the economy.

On the other hand, two recent papers have shown interesting features of economic activity synchronization when the business cycle is disaggregated at the regional level. In the first, Owyang et al. (2005) investigate the evolution of the individual business cycle phases of U.S. states. By following a univariate approach, the authors find that U.S. states differ significantly in the timing of switches between expansions and recessions, and also differ in the extent to which phases in state business cycles are synchronous with those of the national economy. In the second paper, Hamilton and Owyang (2012) use a unified framework to go through the propagation of regional recessions in the United States, using a multivariate approach that focuses on clustering the states that share similar business cycle characteristics. They find that differences across states appear to be a matter of timing and that they can be grouped into three clusters, with some entering recession or recovering before others. Although these previous studies provide useful insights about the overall synchronization pattern in a given sample period, they are not able to detect changes in patterns occurring in these time spans.

This study intends to unify both concepts: first, the dynamic synchronization of pairwise cycles, by using the framework proposed in Section 2; and second, the dynamic interdependence among all U.S. states, by relying on network analysis, in order to assess the presence and the nature of potential changes in the regional propagation of contractionary shocks. For this purpose, I use data on U.S. states coincident indexes, proposed in Crone and Matthews(2005) and provided by the Federal Reserve Bank of Philadelphia, as monthly indicators of the overall economic activity at the state level for the time span August 1979 to March 2013 (Alaska and Hawaii are excluded as in Hamilton and Owyang (2012)). The Chicago Fed National Activity Index (CFNAI) is used as a monthly measure of the U.S. national business cycle. All these indexes of real economic activity, for each state and for the entire United States, have been constructed based on the principle of co-movement among industrial production, employment, sales and income measures. 


\subsection{Bivariate Analysis}

The analysis for 48 states plus the United States as a whole requires the modelling of the $C_{2}^{49}=1,176$ pairwise comparisons. To assess the performance of the proposed Markovswitching synchronization model, two selected examples are analyzed in detail. ${ }^{8}$ The first example focuses on the case of two states that have a high share of national GDP: New York (7.68\%) and Texas (7.95\%). Table 4 reports the Bayesian estimates for the New York vs. Texas model, showing negative growth rates when $S_{t}=0$ and positive growth when $S_{t}=1$, for both states. It is worth highlighting the estimates of the transition probabilities associated with the state variable that measures synchronization, $V_{t}$. The probability of remaining in a regime of high synchronization is almost equal to the probability of remaining in a low synchronization regime, about 0.96. This result is corroborated in Chart A of Figure 3, which plots the probabilities of recession for New York and Texas along with the corresponding time-varying synchronization, $\delta_{t}^{N Y, T X}$, as defined in Equation (16). As can be seen in the top and middle charts, from the 1980s to the mid-1990s, these states experienced recessions at different times. This is reflected in the low values of the synchronicity, plotted at the bottom of Chart A. However, since the mid-1990s, both economies have been experiencing the same recession chronology, which is consistent with the increase in the synchronicity observed after the mid-1990s.

The second example analyzes the case of two states with different shares of GDP: the state with the highest, California (13.34\%); and the state with the lowest, Vermont $(0.18 \%)$. Table 5 presents the Bayesian parameter estimates of the model. Unlike the previous example, in the California vs. Vermont model, the probability of remaining in a high synchronization regime, 0.97 , is higher than the probability of remaining in a low synchronization regime, 0.93. This is also illustrated in Chart B of Figure 3, which shows that, in general, both states have experienced the same business cycle chronology, entering recessions and expansions synchronously, with the exception of one period. Specifically, in 1989, Vermont entered a recessionary phase, while California was still growing until mid1990, when it too started to experience a recession. However, at the beginning of 1992, Vermont started an expansionary phase, while California remained in recession until 1994. These desynchronicities are reflected in the downturn of the dynamic synchronization, $\delta_{t}^{C A, V T}$, during that period, shown in the bottom panel of Chart B.

Considerable heterogeneity was found in the dynamics of the estimated time-varying synchronizations, finding cases involving significant changes, and cases where the synchronization was almost constant, at low or high levels. Although the proposed framework can provide information on the synchronization between any pair of states for any given period of time, other ways to summarize the information are needed, since policy-makers are interested in the "big picture" of the overall regional synchronization path.

\footnotetext{
${ }^{8}$ The results for the other 1,174 cases are available from the author upon request.
} 


\subsection{Multivariate Analysis}

As suggested by Tim (2002) and Camacho et al. (2006), the multi-dimensional scaling (MDS) method is a helpful tool for identifying cyclical affiliations between economies, since it seeks to find a low-dimensional coordinate system to represent $n$-dimensional objects and create a map of lower dimension $(k)$. Traditionally, studies use as input for this method a symmetric matrix, $\Gamma$, that summarizes the cyclical distances between economies for a given time span. Each entry $\gamma^{i j}$ of the matrix assigns a value characterizing the distance between economies $i$ and $j$. The output of the MDS consists of one map showing the general picture for all the cyclical affiliations.

The dynamic synchronization measures obtained in the bivariate analysis, $0 \leq \delta_{t}^{i j} \leq 1$, can be easily converted into desynchronization measures, $\gamma_{t}^{i j}=1-\delta_{t}^{i j}$. Accordingly, $\gamma_{t}^{i j}$ can be interpreted as cyclical distances, allowing the construction of the dissimilarity matrix $\Gamma$, for each time period:

$$
\Gamma_{t}=\left(\begin{array}{ccccc}
1 & \gamma_{t}^{12} & \gamma_{t}^{13} & \ldots & \gamma_{t}^{1 n} \\
\gamma_{t}^{21} & 1 & \gamma_{t}^{23} & \ldots & \gamma_{t}^{2 n} \\
\gamma_{t}^{31} & \gamma_{t}^{32} & 1 & \ldots & \gamma_{t}^{3 n} \\
\vdots & \vdots & \vdots & \ddots & \vdots \\
\gamma_{t}^{n 1} & \gamma_{t}^{n 2} & \gamma_{t}^{n 3} & \ldots & 1
\end{array}\right)
$$

which provides the possibility of assessing changes in the general picture of all cyclical affiliations of U.S. states.

In a recent work on MDS, Xu et al. (2012) propose a way to deal with MDS in a dynamic fashion, where the dimensional coordinates of the projection of any two objects, $i$ and $j$, are computed by minimizing the stress function,

$$
\min _{\tilde{\gamma}_{t}^{i j}}=\frac{\sum_{i=1}^{n} \sum_{j=1}^{n}\left(\gamma_{t}^{i j}-\tilde{\gamma}_{t}^{i j}\right)^{2}}{\sum_{i, i}\left(\gamma_{t}^{i j}\right)^{2}}+\beta \sum_{i=1}^{n} \tilde{\gamma}_{t \mid t-1}^{i},
$$

where

$$
\begin{aligned}
\tilde{\gamma}_{t}^{i j} & =\left(\left\|z_{i, t}-z_{j, t}\right\|^{2}\right)^{1 / 2} \\
\tilde{\gamma}_{t \mid t-1}^{i} & =\left(\left\|z_{i, t}-z_{i, t-1}\right\|^{2}\right)^{1 / 2}
\end{aligned}
$$

$z_{i, t}$ and $z_{j, t}$ are the $k$-dimensional projection of the objects $i$ and $j$, and $\beta$ is a temporal regularization parameter that serves to zoom in or zoom out changes between frames at $t$ and at $t+1$, always keeping the same dynamics independent of its value. In principle, $\beta$ can be simply set up to 1 ; however, since the data in $\Gamma_{t}$ belong to the unit interval, for a 
more adequate visual perception of the transitions between frames it is set up to 0.1. The output of the minimization in Equation (48) provides a two-dimensional representation of $\Gamma_{t}$.

The synchronization maps of U.S. states for the first month of the last four recessions are plotted in the charts of Figure 4. Each point in the charts represents a state, and the middle point refers to the United States as a whole. The closeness between two points in the plane refers to their degree of synchronicity, i.e., the closer the points are, the greater their synchronization. The figure corroborates the premise in the introduction of this paper about the existence of significant changes in the grouping pattern among regional economies over time. Specifically, the top-left chart plots the scenario for the 1981 recession, where a large number of states were synchronized with each other, while the remaining states, such as Florida, Colorado, Texas, North Dakota, West Virginia, among others, were following independent patterns. Also, notice that states such as Nevada, North Carolina, Vermont and Tennessee, were the ones more in sync with the U.S. business cycle during that month. The top-right corner presents the situation for the 1990 recession, showing a different grouping pattern, characterized by one large group of states in sync with each other and two small clusters, the first one composed of New Hampshire, Massachusetts, Connecticut, Vermont, New Jersey, Maine and Rhode Island, and the second one composed of New York, Virginia, Delaware and Maryland. Notice that in this month, states such as Florida, Pennsylvania and California, among others, were the ones more in sync with the U.S. cycle. The bottom charts present the scenarios for the 2001 and 2007 recessions, in the left and right corner respectively. Again, the pattern changed with respect to the previous episodes, since the last two recessions were characterized by a core (composed of states highly in sync) and periphery (composed of independent states) structure. Notice that the core during the 2001 recession is tighter than the core during the 2007 recession. The full animated representation can be found at the author's webpage. ${ }^{9}$

An additional advantage of the proposed framework is the possibility of recovering the stationary measures of synchronization, by using the ergodic probabilities associated with the latent variable $V_{t}$. Chart A of Figure 5 plots the stationary grouping pattern, which can be interpreted as the average pattern from August 1979 to March 2013. It shows three groups of states: one is close to the U.S. cycle, the second is less but still close to the U.S. cycle, while the third is characterized by the states following independent dynamics. To assess whether this result reconciles with the one in Hamilton and Owyang (2012), Chart B of Figure 5 plots the clusters obtained by those authors, clearly finding that both results coincide, not only in the number of clusters but also in the states that correspond to each cluster. Moreover, this result is not only robust to the methodology employed, but also to the data used, since Hamilton and Owyang (2012) use annualized quarter-to-

\footnotetext{
${ }^{9}$ https://sites.google.com/site/daniloleivaleon/media
} 
quarter growth rates of payroll employment, while I use monthly growth rates of state coincident indexes of economic activity. These facts show one of the main contributions of the proposed framework, which is to provide synchronization measures that may change over time, and that can be collapsed into ergodic measures that yield results consistent with those in previous work.

Regarding the cyclical relationship between states and the national business cycle, Table 6 reports the corresponding ergodic synchronizations, showing the range from the highest, which is North Carolina with 0.91, to the lowest one, Oklahoma with 0.19, and revealing that states with the highest GDP share do not necessarily represent the states showing the highest synchronicity with the national business cycle. To provide a visual perspective, Chart A of Figure 6 plots a U.S. map with the estimates obtained in this paper, and Chart B plots the concordance pattern obtained in Owyang et al. (2005) by calculating the percentage of the time two economies were in the same regime, based on univariate MS models for each state. Although both results report high values in most of the states located in the east region and medium values in a few states located in the west, the stationary synchronization measure presents higher dispersion than the concordance, as can be seen in the associated histograms. This comparison helps to disentangle in a more precise way the cyclical relationship between the business cycles of states and the nation.

\subsection{Network Analysis}

Recent works by Carvalho (2008), Gabaix (2011), Acemoglu et al. (2012), among others, rely on network analysis to show how idiosyncratic shocks, at the firm or sectoral level, may originate macroeconomic fluctuations, given their interlinkages. Although, such analysis primarily relies on the economy's sectoral disaggregation, it may be interesting to assess if another type of disaggregation, e.g., regional, may also have significant implications on aggregate fluctuations.

The intuition behind the synchronization measure in Equation (16) relies on the fact that if $\delta_{t}^{i j}$ is close to 1 , it is likely that at time $t$, economies $i$ and $j$ are sharing the same business cycle phases, creating a link of interdependence between them. On the other hand, if $\delta_{t}^{i j}$ is close to 0 , it means that the economies are following independent phases and thus are not linked. ${ }^{10}$ Therefore, by letting $H=\left\{h_{i}\right\}_{1}^{n}$ be the set of $n$ economies taking the interpretation of nodes, $h_{i}$ for $i=1, \ldots, n$, and defining $\delta_{t}^{i j}$ as the probability that nodes $h_{i}$ and $h_{j}$ are linked at time $t$, the matrix $\Delta_{t}=\mathbf{1}_{n}-\Gamma_{t}$, can be interpreted

\footnotetext{
${ }^{10}$ Notice that the proposed synchronization modelling approach distinguishes between the state in which two economies are in recession but their cycles are independent and just coincided, from the state where the two economies are in recession because they are under a regime of dependence, i.e., states 1 and 5 of $S_{a b, t}^{*}$ in Equation (11), respectively.
} 
as a weighted network of synchronization with Markovian dynamics. ${ }^{11}$ Consequently, the cyclical interdependence of a large set of economies can be dynamically assessed under a unified framework by relying on network analysis. It is worth noting that although the construction of $\Delta_{t}$ requires the computation of several bivariate models of the type in Equation (4), it may be less restrictive and involve less parameter and regime uncertainty than the computation of a framework with a similar non-linear nature but involving all $n$ economies simultaneously. However, further research in this respect is needed.

To provide a glimpse of the shape that the Markov-switching synchronization network (MSYN) has taken during contractionary episodes, the charts of Figure 7 plot the corresponding network graph for the first month of the last four recessions. Given that the MSYN is a weighted network, in order to make the graphical representation possible, a link between nodes $i$ and $j$ is plotted if $\delta_{t}^{i j}>0.5$; otherwise, no link is plotted between them. The figure corroborates the grouping pattern of one big cluster and independent states in the 1981 recession, some small clusters in the 1990 recession and a core and periphery structure in the 2001 and 2007 recessions, with a more concentrated core in the most recent recession. ${ }^{12}$

The main advantage of providing a network analysis for the present framework is that all the information on synchronicities in the current analysis can be summarized in just one measure, the closeness centrality. There are several measures regarding the centrality of a network, but given that desynchronization measures are interpreted as distances, the most appropriate one for this context is the closeness centrality.

For robustness purposes, two variations of the closeness centrality are analyzed in this section. For each of them, it is necessary to first compute the centrality of each node,

$$
C_{t}(i)=\frac{1}{\sum_{j \neq i \mid t} d_{t}(i, j)}, \text { for } i=1,2, \ldots, n,
$$

where $d(i, j)$ is the length of the shortest path between nodes $i$ and $j$, which can be computed by the Dijkstra (1959) algorithm. ${ }^{13}$ Thus, the more central a node is, the lower the total distance from it to all other nodes. Closeness can be regarded as a measure of how fast it will take to spread information, e.g., risk, economic shocks, etc., from node $i$ to all other nodes sequentially. For an overview of definitions in network analysis, see Goyal (2007).

Once the dynamic centrality of each node has been computed, the information about

\footnotetext{
${ }^{11}$ The term $\mathbf{1}_{n}$ represents a squared matrix of size $n$ with all entries equal to 1 .

${ }^{12}$ Notice that, although the U.S. business cycle is not included in the network analysis, only those of the states, each chart in the figure shows a close relation with the corresponding one in Figure 4.

${ }^{13}$ For example, in a set $H^{\prime}=\{a, b, c\}$ where the distances $\gamma=1-\delta$ are given by $\gamma^{a b}=0.5, \gamma^{a c}=0.9$ and $\gamma^{b c}=0.2$, the shortest path between $a$ and $c$ will be 0.7 , since $\gamma^{a b}+\gamma^{b c}<\gamma^{a c}$. Thus, notice that $d(a, c)$ does not necessarily have to be equal to $\gamma^{a c}$.
} 
the whole network's centrality can be typically assessed as follows:

$$
C_{t}^{N}=\sum_{i=1 \mid t}^{k}\left[C_{t}\left(i^{*}\right)-C_{t}(i)\right]
$$

where $i^{*}$ is the node that attains the highest closeness centrality across all nodes at time $t$. The second measure, consists on the average across all nodes' centralities, $C_{t}(i)$, defined by

$$
C_{t}^{A}=\sum_{i=1 \mid t}^{k} C_{t}(i) .
$$

These two measures, which provide information on the changes in the degree of aggregate synchronization among the economies in the set $H$, for the present case between the states of the United States, can be used to investigate the relationship between regional business cycle interdependence and aggregate fluctuations. ${ }^{14}$

One of the main findings in Hamilton and Owyang (2012) is the substantial heterogeneity across regional recessions in the United States at the state level. How such heterogeneity could change over time, however, is an issue that has remained uninvestigated. The proposed framework is used to dynamically quantify the substantial regional heterogeneity under the unified setting MSYN. The intuition behind the state's centrality in Equation (51) is the following: if, at time $t$, state $i$ is highly synchronized with respect to the rest of U.S. states, its total distance from them, $\sum_{j \neq i \mid t} d_{t}(i, j)$, would tend to be low and its centrality, $C_{t}(i)$, to be high. If a similar behaviour occurs with the remaining $n-1$ states, the MSYN's centrality would also tend to take high values. This means that high global interdependence, or, equivalently, high homogeneity of regional recessions, is associated with high values of the MSYN's centrality $C_{t}^{\Upsilon}$, for $\Upsilon=N, A$.

Chart A of Figure 8 plots the network centrality, $C_{t}^{N}$, and the average centrality, $C_{t}^{A}$, in standardized terms to facilitate their comparison. Both measures show similar dynamics, experiencing substantial changes over time that have a close relation with the national recessions dated by the NBER, and showing some interesting features. First, the centrality shows a markedly high tendency to increase some months before national recessions take place and to maintain high values during the whole contractionary episode, implying that sudden increases in the degree of interdependence among states may be useful to signal upcoming national recessions.

Second, once national recessions have ended, the centrality remains high for some period of time. This is because the whole economy is recovering from the recession and most of the states are synchronized, although, this time, in a recovery regime. Notice that

\footnotetext{
${ }^{14} \mathrm{~A}$ third measure was also computed by extracting the common component among the nodes' centralities using principal component analysis. However, the results were similar to those of obtained with the average centrality. Therefore, they are not shown.
} 
the highest interdependence level, occurring in October 2003, roughly coincided with the highest growth rate of real GDP experienced by the U.S. economy from the end of 2000 until the present time.

Third, after this phase of recovery has ended and the U.S. economy starts its moderated expansionary path, the centrality decreases until it reaches a certain stable level, which prevails until another recession takes place and the cycle repeats. Notice that the periods with higher heterogeneity across regional business cycles do not occur during recessions or recoveries, but during periods of stable economic expansion. These three observations reveal that regional economies in the United States at the state level are subject to cycles of interdependence that are highly associated with the national business cycle.

Fourth, the centrality measures during the past two national recessions were almost twice as high as during the previous recessions, corroborating the core-periphery structure observed in the MDS analysis for the corresponding periods and plotted in the bottom charts of Figure 7. This result discloses a change in the propagation pattern of aggregate recessionary shocks. On the one hand, during the pre-2000 recessions, those shocks were spread mainly toward a few large states, in terms of share of GDP, such as California, Georgia, Massachusetts, New Jersey and New York during the 1981 recession, and Florida, Georgia, North Carolina and Pennsylvania during the 1990 recession. On the other hand, during the post-2000 recessions, such shocks were more uniformly and synchronously distributed across states, in particular, to the ones in the core that were the majority, as can be seen in the charts of Figure 4. For robustness purposes, the centrality measures were also computed using the filtered, instead of the smoothed, probabilities of $V_{t}$ which are plotted in Chart B of Figure 8. They show essentially the same results.

Finally, to address changes in the clustering pattern in a statistical rather than visual manner, I compute the clustering coefficient of the MSYN for every time period by following Strogatz and Watts (1998), which allows the measurement of the level of cohesiveness between the business cycle phases of U.S. states. The dynamic clustering coefficient is plotted in Figure 9, showing relatively low values during the 1981 and 1990 recessions and high values during the 2001 and 2007 recessions. Moreover, it shows that in the mid-1990s there was a significant change in the regional cohesiveness. Before that time, the clustering coefficient followed short cycles, but after the mid-1990s, it remained almost stable at higher values, corroborating the change in the propagation of contractionary shocks that occurred since the 2001 recession and providing evidence that the U.S. economy's regions have become more interdependent since the early 1990s.

There are several potential channels driving this change, such as macroeconomic or financial factors. Further research on these issues is required. 


\section{Conclusions}

Most of the studies on business cycle synchronization provide a general pattern of cyclical affiliations between economies for a given time span. However, little has been done to assess potential pattern changes that may occur during such a time span. This paper proposed an extended Markov-switching framework to assess changes in the synchronization of cycles by inferring the time-varying dependency relationship between the latent variables governing Markov-switching models. The reliability of the approach to track synchronization changes is confirmed by Monte Carlo experiments.

The proposed framework is applied to investigate potential variations in the cyclical interdependence between the states of the United States. There are three main findings. First, the results report the existence of interdependence cycles that are associated with NBER recessions. Such cycles are defined as periods characterized by low cyclical heterogeneity across states, experienced during the recessionary and recovery phases, followed by longer periods of high cyclical heterogeneity that occur during the phases of stable growth. Second, there are substantial variations in the grouping pattern of states over time that can be monitored on a monthly basis, ranging from a scheme characterized by several clusters of states to a core and periphery structure, composed of highly and lowly synchronized states, respectively. Third, there is evidence of a change in the propagation pattern of recessionary shocks across states. Up to the 1991 recession, recessionary shocks were spread mainly toward a few large states, in terms of share of GDP. But after that, contractionary shocks were more synchronously and uniformly spread toward most of the U.S. states, implying that U.S. regions have become more interdependent since the early 1990s. 


\section{Appendix}

\section{A Bayesian Parameter Estimation}

The approach to estimate $\theta$ relies on a bivariate extended version of the multi-move Gibbssampling procedure implemented by Kim and Nelson (1999) for Bayesian estimation of univariate Markov-switching models. In this setting, both the parameters of the model $\theta$ and the Markov-switching variables $\tilde{S}_{k, T}=\left\{S_{k, t}\right\}_{1}^{T}$ for $k=a, b, \tilde{S}_{T}=\left\{S_{t}\right\}_{1}^{T}$ and $\tilde{V}_{T}=\left\{V_{t}\right\}_{1}^{T}$ are treated as random variables given the data in $\tilde{y}_{T}=\left\{y_{t}\right\}_{1}^{T}$. The purpose of this Markov chain Monte Carlo simulation method is to approximate the joint and marginal distributions of these random variables by sampling from conditional distributions.

\section{A.1 Priors}

For the mean and variance parameters in vector $\theta$, the independent Normal-Wishart prior distribution is used:

$$
p\left(\mu, \Sigma^{-1}\right)=p(\mu) p\left(\Sigma^{-1}\right),
$$

where

$$
\begin{aligned}
\mu & \sim N\left(\underline{\mu}, \underline{V}_{\mu}\right) \\
\Sigma^{-1} & \sim W\left(\underline{S}^{-1}, \underline{v}\right),
\end{aligned}
$$

and the associated hyperparameters are given by $\underline{\mu}=(-1,2-1,2)^{\prime}, \underline{V}_{\mu}=I, \underline{S}^{-1}=I$, $\underline{v}=0$.

For the transition probabilities $p_{a, 00}, p_{a, 11}$, from $S_{a, t}, p_{b, 00}, p_{b, 11}$, from $S_{b, t}, p_{00}, p_{11}$, from $S_{t}$; and $p_{v, 00}, p_{v, 11}$ from $V_{t}$, Beta distributions are used as conjugate priors:

$$
\begin{aligned}
p_{k, 00} & \sim B e\left(u_{k, 11}, u_{k, 10}\right), p_{k, 11} \sim B e\left(u_{k, 00}, u_{k, 01}\right), \text { for } k=a, b \\
p_{v, 00} & \sim B e\left(u_{v, 11}, u_{v, 10}\right), p_{v, 11} \sim B e\left(u_{v, 00}, u_{v, 01}\right), \\
p_{00} & \sim B e\left(u_{11}, u_{10}\right), p_{11} \sim B e\left(u_{00}, u_{01}\right),
\end{aligned}
$$

where the hyperparameters are given by $u_{\iota, 01}=2, u_{\iota, 00}=8, u_{\iota, 10}=1$ and $u_{\iota, 11}=9$, for $\iota=a, b, v,_{-}$. For each pairwise model, 6,000 iterations were performed, with the first 1,000 discarded.

\section{A.2 Drawing $\tilde{S}_{a, T}, \tilde{S}_{b, T}, \tilde{S}_{T}$ and $\tilde{V}_{T}$ given $\theta$ and $\tilde{y}_{T}$}

Following the result in Equation (15), in order to make inferences on the bivariate dynamics of the model in Equation (4) driven by $\tilde{S}_{a b, T}=\left\{S_{a b, t}\right\}_{1}^{T}$ and described in Equation (6), 
it is only necessary to make inferences on the dynamics of the single-state variables $\tilde{S}_{a, T}$, $\tilde{S}_{b, T}, \tilde{S}_{T}$ and $\tilde{V}_{T}$. This can be done following the results in Kim and Nelson (1999) by first computing draws from the conditional distributions:

$$
\begin{aligned}
g\left(\tilde{S}_{k, T} \mid \theta, \tilde{y}_{T}\right) & =g\left(S_{k, T} \mid \tilde{y}_{T}\right) \prod_{t=1}^{T} g\left(S_{k, t} \mid S_{k, t+1}, \tilde{y}_{t}\right), \text { for } k=a, b \\
g\left(\tilde{S}_{T} \mid \theta, \tilde{y}_{T}\right) & =g\left(S_{T} \mid \tilde{y}_{T}\right) \prod_{t=1}^{T} g\left(S_{t} \mid S_{t+1}, \tilde{y}_{t}\right) \\
g\left(\tilde{V}_{T} \mid \theta, \tilde{y}_{T}\right) & =g\left(V_{T} \mid \tilde{y}_{T}\right) \prod_{t=1}^{T} g\left(V_{t} \mid V_{t+1}, \tilde{y}_{t}\right)
\end{aligned}
$$

In order to obtain the two terms in the right-hand side of Equations (58) and (59), the following two steps can be employed:

Step 1: The first term can be obtained by running the filtering algorithm developed in Section 2.1, to compute $g\left(\tilde{S}_{k, t} \mid \tilde{y}_{t}\right)$ for $k=a, b, g\left(\tilde{S}_{t} \mid \tilde{y}_{t}\right)$ and $g\left(\tilde{V}_{k, t} \mid \tilde{y}_{t}\right)$ for $t=1,2, \ldots, T$, saving them and taking the elements for which $t=T$.

Step 2: The product in the second term can be obtained for $t=T-1, T-2, \ldots, 1$, by following the result:

$$
\begin{aligned}
g\left(S_{t} \mid \tilde{y}_{t}, S_{t+1}\right) & =\frac{g\left(S_{t}, S_{t+1} \mid \tilde{y}_{t}\right)}{g\left(S_{t+1} \mid \tilde{y}_{t}\right)} \\
& \propto g\left(S_{t+1} \mid S_{t}\right) g\left(S_{t} \mid \tilde{y}_{t}\right),
\end{aligned}
$$

where $g\left(S_{t+1} \mid S_{t}\right)$ corresponds to the transition probabilities of $S_{t}$ and $g\left(S_{t} \mid \tilde{y}_{t}\right)$ that were saved in Step 1.

Then, it is possible to compute

$$
\operatorname{Pr}\left[S_{t}=1 \mid S_{t+1}, \tilde{y}_{t}\right]=\frac{g\left(S_{t+1} \mid S_{t}=1\right) g\left(S_{t}=1 \mid \tilde{y}_{t}\right)}{\sum_{j=0}^{1} g\left(S_{t+1} \mid S_{t}=j\right) g\left(S_{t}=j \mid \tilde{y}_{t}\right)}
$$

and generate a random number from a $U[0,1]$. If that number is less than or equal to $\operatorname{Pr}\left[S_{t}=1 \mid S_{t+1}, \tilde{y}_{t}\right]$, then $S_{t}=1$, otherwise $S_{t}=0$. The same procedure applies for $S_{a, t}$, $S_{b, t}$ and $V_{t}$, and, by using Equation (15), inference of $\tilde{S}_{a b, T}$ can be done.

\section{A.3 Drawing $p_{a, 00}, p_{a, 11}, p_{b, 00}, p_{b, 11}, p_{00}, p_{11}, p_{v, 00}, p_{v, 11}$ given $\tilde{S}_{a, T}, \tilde{S}_{a, T}, \tilde{S}_{T}$ and $\tilde{V}_{T}$}

Conditional on $\tilde{S}_{k, T}$ for $k=a, b, \tilde{S}_{T}$ and $\tilde{V}_{T}$, the transition probabilities are independent from the data set and the model's parameters. Hence, focusing on the case of $\tilde{S}_{T}$, the likelihood function of $p_{00}, p_{11}$ is given by

$$
L\left(p_{00}, p_{11} \mid \tilde{S}_{T}\right)=p_{00}^{n_{00}}\left(1-p_{00}^{n_{01}}\right) p_{11}^{n_{11}}\left(1-p_{11}^{n_{10}}\right),
$$


where $n_{i j}$ refers to the transitions from state $i$ to $j$, accounted for in $\tilde{S}_{T}$.

Combining the prior distribution in Equation (57) with the likelihood, the posterior distribution is given by

$$
p\left(p_{00}, p_{11} \mid \tilde{S}_{T}\right) \propto p_{00}^{u_{00}+n_{00}-1}\left(1-p_{00}\right)^{u_{01}+n_{01}-1} p_{11}^{u_{11}+n_{11}-1}\left(1-p_{11}\right)^{u_{10}+n_{10}-1},
$$

which indicates that draws of the transition probabilities will be taken from

$$
p_{00}\left|\tilde{S}_{T} \sim B e\left(u_{00}+n_{00}, u_{01}+n_{01}\right), p_{11}\right| \tilde{S}_{T} \sim B e\left(u_{11}+n_{11}, u_{10}+n_{10}\right) .
$$

The same procedure applies for the cases of $\tilde{S}_{k, T}$ for $k=a, b$ and $\tilde{V}_{T}$.

A.4 Drawing $\mu_{0, a}, \mu_{1, a}, \mu_{0, b}, \mu_{1, b}$ given $\sigma_{a}^{2}, \sigma_{b}^{2}, \sigma_{a b}, \tilde{S}_{a, T}, \tilde{S}_{b, T}, \tilde{S}_{T}, \tilde{V}_{T}$ and $\tilde{y}_{T}$

The model in Equation (4) can be compactly expressed as

$$
\begin{aligned}
{\left[\begin{array}{l}
y_{a, t} \\
y_{b, t}
\end{array}\right] } & =\left[\begin{array}{cccc}
1 & S_{a, t} & 0 & 0 \\
0 & 0 & 1 & S_{b, t}
\end{array}\right]\left[\begin{array}{l}
\mu_{a, 0} \\
\mu_{a, 1} \\
\mu_{b, 0} \\
\mu_{b, 1}
\end{array}\right]+\left[\begin{array}{l}
\varepsilon_{a, t} \\
\varepsilon_{b, t}
\end{array}\right],\left[\begin{array}{l}
\varepsilon_{a, t} \\
\varepsilon_{b, t}
\end{array}\right] \sim N\left(\left[\begin{array}{l}
0 \\
0
\end{array}\right],\left[\begin{array}{cc}
\sigma_{a}^{2} & \sigma_{a b} \\
\sigma_{a b} & \sigma_{b}^{2}
\end{array}\right]\right) \\
y_{t} & =\bar{S}_{t} \mu+\xi_{t}, \quad \xi_{t} \sim N(\mathbf{0}, \Sigma),
\end{aligned}
$$

stacking as

$$
y=\left[\begin{array}{c}
y_{1} \\
y_{2} \\
\vdots \\
y_{T}
\end{array}\right], \bar{S}=\left[\begin{array}{c}
\bar{S}_{1} \\
\bar{S}_{2} \\
\vdots \\
\bar{S}_{T}
\end{array}\right] \text {, and } \xi=\left[\begin{array}{c}
\xi_{1} \\
\xi_{2} \\
\vdots \\
\xi_{T}
\end{array}\right]
$$

The model in Equation (66) remains written as a normal linear regression model with an error covariance matrix of a particular form:

$$
y=S \mu+\xi, \quad \xi \sim N(\mathbf{0}, I \otimes \Sigma)
$$

Conditional on the covariance matrix parameters, state variables and the data, by using the corresponding likelihood function, the conditional posterior distribution

$p\left(\mu \mid \tilde{S}_{a, T}, \tilde{S}_{b, T}, \tilde{S}_{T}, \tilde{V}_{T}, \Sigma^{-1}, \tilde{y}_{T}\right)$ takes the form

$$
\mu \mid \tilde{S}_{a, T}, \tilde{S}_{b, T}, \tilde{S}_{T}, \tilde{V}_{T}, \Sigma^{-1}, \tilde{y}_{T} \sim N\left(\bar{\mu}, \bar{V}_{\mu}\right),
$$


where

$$
\begin{aligned}
\bar{V}_{\mu} & =\left(\underline{V}_{\mu}^{-1}+\sum_{t=1}^{T} \bar{S}_{t}^{\prime} \Sigma^{-1} \bar{S}_{t}\right)^{-1} \\
\bar{\mu} & =\bar{V}_{\mu}\left(\underline{V}_{\mu}^{-1} \underline{\mu}+\sum_{t=1}^{T} \bar{S}_{t}^{\prime} \Sigma^{-1} y_{t}\right) .
\end{aligned}
$$

After drawing $\mu=\left(\mu_{a, 0}, \mu_{a, 1}, \mu_{b, 0}, \mu_{b, 1}\right)^{\prime}$ from the above multivariate distribution, if the generated value of $\mu_{a, 1}$ or $\mu_{b, 1}$ is less than or equal to 0 , that draw is discarded; otherwise, it is saved, in order to ensure that $\mu_{a, 1}>0$ and $\mu_{b, 1}>0$.

\section{A.5 Drawing $\sigma_{a}^{2}, \sigma_{b}^{2}, \sigma_{a b}$ given $\mu_{0, a}, \mu_{1, a}, \mu_{0, b}, \mu_{1, b}, \tilde{S}_{a, T}, \tilde{S}_{b, T}, \tilde{S}_{T}, \tilde{V}_{T}$ and $\tilde{y}_{T}$}

Conditional on the mean parameters, state variables and the data, by using the corresponding likelihood function, the conditional posterior distribution

$$
p\left(\Sigma^{-1} \mid \tilde{S}_{a, T}, \tilde{S}_{b, T}, \tilde{S}_{T}, \tilde{V}_{T}, \mu, \tilde{y}_{T}\right)
$$

takes the form

$$
\Sigma^{-1} \mid \tilde{S}_{a, T}, \tilde{S}_{b, T}, \tilde{S}_{T}, \tilde{V}_{T}, \mu, \tilde{y}_{T} \sim W\left(\bar{S}^{-1}, \bar{v}\right),
$$

where

$$
\begin{aligned}
\bar{v} & =T+\underline{v} \\
\bar{S} & =\underline{S}+\sum_{t=1}^{T}\left(y_{t}-\bar{S}_{t} \mu\right)\left(y_{t}-\bar{S}_{t} \mu\right)^{\prime} .
\end{aligned}
$$

After $\Sigma^{-1}$ is generated, the elements in $\Sigma$ are recovered. 


\section{References}

[1] Acemoglu, D, V. M. Carvalho, A. Ozdaglar and A. Tahbaz-Salehi (2012), The network origins of aggregate fluctuations. Econometrica 80:5, 1977-2016.

[2] Anas, J., M. Billio, L. Ferrara and M. Lo Duca (2007), Business Cycle Analysis with Multivariate Markov Switching Models. Working Papers, Department of Economics, University of Venice Ca' Foscari.

[3] Artis, M., M. Marcellino and T. Proietti (2004), Dating Business Cycles: A Methodological Contribution with an Application to the Euro Area. Oxford Bulletin of Economics and Statistics 66:4, 537-565.

[4] Bai J, P. Wang, (2011), Conditional Markov chain and its application in economic time series analysis. Journal of Applied Econometrics 26:5, 715-734.

[5] Bengoechea, P., M. Camacho and G. Perez-Quiros (2006), A useful tool for forecasting the Euro-area business cycle phases. International Journal of Forecasting 22:4, 735749 .

[6] Boldin, M D. (1996), A Check on the Robustness of Hamilton's Markov Switching Model Approach to the Economic Analysis of the Business Cycle. Studies in Nonlinear Dynamics and Econometrics 1:1, 35-46.

[7] Cakmakli, C, R. Paap and D. Van Dijk (2011), Modeling and Estimation of Synchronization in Multistate Markov. Tinbergen Institute Discussion Papers 11-002/4.

[8] Camacho, M. and G. Perez-Quiros (2006), A new framework to analyze business cycle synchronization. Nonlinear Time Series Analysis of Business Cycles. Elsevier's Contributions to Economic Analysis series. Chapter 5, 276, 133-149.

[9] Camacho, M., G. Perez-Quiros and L. Saiz (2006), Are European business cycles close enough to be just one? Journal of Economic Dynamics and Control 30:9-10, $1687-1706$.

[10] Camacho, M., and G. Perez-Quiros (2007), Jump-and-rest effect of U.S. business cycles. Studies in Nonlinear Dynamics and Econometrics Vol. 11: No. 4, 3.

[11] Carvalho, V. M. (2008), Aggregate Fluctuations and the Network Structure of Intersectoral Trade. Working Paper, CREI, 1977-2004.

[12] Chauvet, M and Z. Senyuz (2012). A Joint Dynamic Bi-Factor Model of the Yield Curve and the Economy as a Predictor of Business Cycles. Finance and Economics Discussion Series 32, Federal Reserve Board. 
[13] Crone, T. M. and C. Matthews (2005), Consistent Economic Indexes for the 50 States. Review of Economics and Statistics 87:4, 593-603.

[14] Dijkstra, E. W. (1959), A note on two problems in connexion with graphs. Numerische Mathematik 1 269-271.

[15] Gabaix, X. (2011), The Granular Origins of Aggregate Fluctuations. Econometrica $79,733-772$.

[16] Goyal, S. (2007), Connections: An Introduction to the Economics of Networks. Princeton University Press.

[17] Guha, D, and A. Banerji (1998), Testing for cycles: A Markov switching approach. Journal of Economic and Social Measurement 25: 163-182.

[18] Hamilton, J. D. (1989), A new approach to the economic analysis of nonstationary time series and the business cycle. Econometrica 57:2, 357-384.

[19] Hamilton, J. D. (1994), Time Series Analysis. Princeton, NJ: Princeton University Press.

[20] Hamilton, J. D and G. Lin (1996), Stock Market Volatility and the Business Cycle. Journal of Applied Econometrics 11:5, 573-593.

[21] Hamilton, J. D and G. Perez-Quiros (1996), What Do the Leading Indicators Lead? Journal of Business 69:1, 27-49.

[22] Hamilton, J. D. and M. Owyang (2012), The Propagation of Regional Recessions. Review of Economics and Statistics 94:4, 935-947.

[23] Harding, D. and A. Pagan (2006), Synchronization of cycles. Journal of Econometrics 132:1, 59-79.

[24] Kim, C, C.R. Nelson (1999), State-Space Models with Regime Switching: Clasical and Gibbs-Sampling Approaches with Applications. MIT press.

[25] Krolzing, H. (1997), Markov-switching vector autorregresions. Modelling, statistical inference and applications to business cycle analysis. Lecture Notes in Economics and Mathematical Systems 454.

[26] Leiva-Leon, D. (2014), Real vs. Nominal Cycles: A Multistate Markov-Switching BiFactor Approcah. Studies on Nonlinear Dynamics and Econometrics. Forthcoming.

[27] Owyang, M., J. Piger and H. Wall (2005), Business Cycle Phases in U.S. States. Review of Economics and Statistics 87:4, 604-616. 
[28] Pesaran, M. H. and A. Timmermann (2009), Testing Dependence Among Serially Correlated Multicategory Variables. Journal of the American Statistical Association 104:485, 325-337.

[29] Phillips, K. (1991), A two-country model of stochastic output with changes in regime. Journal of International Economics 31: 121-142.

[30] Sims, C. A., and T. Zha (2006), Were There Regime Switches in U.S. Monetary Policy? American Economic Review 96(1): 54-81.

[31] Sims, C. A., D. F. Waggoner and T. Zha (2008), Methods for inference in large multiple-equation Markov-switching models. Journal of Econometrics. 146: 2, 255274 .

[32] Smith, P. A. and P. M. Summers (2005), How well do Markov switching models describe actual business cycles? The case of synchronization. Journal of Applied Econometrics 20:2, 253-274.

[33] Strogatz, S. H. and D. J. Watts (1998), Collective dynamics of 'small-world' networks, Nature 393:6684, 440-442.

[34] Tim, N. H. (2002) Applied Multivariate Analysis. Springer texts in Statistics.

[35] Xu, K. S., M. Kliger and A. O. Hero III (2012), A regularized graph layout framework for dynamic network visualization. Data Mining and Knowledge Discovery 27:1, 84116. 
Table 1: Parameter values for generating processes

\begin{tabular}{cc|cc}
\hline Parameter & Value & Parameter & Value \\
\hline$\mu_{a, 0}^{*}$ & -1 & $\mu_{b, 0}^{*}$ & -2 \\
$\mu_{a, 1}^{*}$ & 2 & $\mu_{b, 1}^{*}$ & 4 \\
$p_{a, 11}^{*}$ & 0.9 & $p_{b, 11}^{*}$ & 0.9 \\
$p_{a, 00}^{*}$ & 0.8 & $p_{b, 00}$ & 0.8 \\
$p_{11}^{*}$ & 0.9 & $p_{V, 11}^{*}$ & 0.9 \\
$p_{00}^{*}$ & 0.8 & $p_{V, 00}^{*}$ & 0.8 \\
$\sigma_{a}^{*}$ & 1 & $\sigma_{b}^{*}$ & 1 \\
$\sigma_{a, b}^{*}$ & 0.1 & & \\
\hline
\end{tabular}

Note: The table shows the parameter values used to generate the stochastic processes $y_{t}$ in Equation (42) for the simulation study in Section 2.2.

Table 2: Performance of parameter estimations

\begin{tabular}{lcccccc}
\hline & $z=1$ & $z=2$ & $z=3$ & $z=4$ & $z=5$ & $z=f\left(V_{t}\right)$ \\
\hline$\mu_{a, 0}^{*}$ & -0.95143 & -0.93985 & -0.94558 & -0.93750 & -0.93166 & -0.93896 \\
$\mu_{a, 1}^{*}$ & 1.91921 & 1.89427 & 1.90213 & 1.89147 & 1.88822 & 1.89089 \\
$p_{a, 11}^{*}$ & 0.89813 & 0.89689 & 0.89729 & 0.89606 & 0.89488 & 0.87803 \\
$p_{a, 00}^{*}$ & 0.79663 & 0.79508 & 0.79393 & 0.78997 & 0.78900 & 0.75626 \\
$\mu_{b, 0}^{*}$ & -1.98915 & -1.99861 & -1.99591 & -1.99903 & -1.99503 & -1.99729 \\
$\mu_{b, 1}^{*}$ & 3.98711 & 3.99662 & 3.99139 & 3.99859 & 3.99011 & 3.99148 \\
$p_{b, 11}^{*}$ & 0.89700 & 0.89576 & 0.89581 & 0.89341 & 0.89188 & 0.86977 \\
$p_{b, 00}$ & 0.79166 & 0.79155 & 0.79088 & 0.78574 & 0.78600 & 0.74422 \\
$p_{11}^{*}$ & 0.89433 & 0.89241 & 0.89406 & 0.89026 & 0.88991 & 0.86936 \\
$p_{00}^{*}$ & 0.78479 & 0.78295 & 0.78600 & 0.77786 & 0.78900 & 0.74178 \\
$p_{V, 11}^{*}$ & - & - & - & - & - & 0.89682 \\
$p_{V, 00}^{*}$ & - & - & - & - & - & 0.80971 \\
\hline
\end{tabular}

Note: The entries in the table report the average of the estimated parameter values through the 1,000 replications for different numbers of synchronization changes, $z$.

Table 3: Performance of regime inferences

\begin{tabular}{lcccccc}
\hline & $z=1$ & $z=2$ & $z=3$ & $z=4$ & $z=5$ & $z=f\left(V_{t}\right)$ \\
\hline$Q P S\left(S_{a, t}\right)$ & 0.05118 & 0.06448 & 0.05571 & 0.06470 & 0.06023 & 0.06074 \\
$Q P S\left(S_{b, t}\right)$ & 0.00749 & 0.00765 & 0.00763 & 0.00829 & 0.00805 & 0.00990 \\
$Q P S\left(V_{t}\right)$ & 0.06387 & 0.08554 & 0.09526 & 0.10988 & 0.11575 & 0.17769 \\
\hline
\end{tabular}

Note: The entries in the table report the average of the Quadratic Probability Score associated with the state variables through the 1,000 replications for different numbers of synchronization changes, $z$. 
Table 4: Dynamic synchronization estimates between New York and Texas

\begin{tabular}{crcr}
\hline & Mean & Std. Dev. & Median \\
\hline$\mu_{n y, 0}$ & -0.12945 & 0.01856 & -0.12895 \\
$\mu_{n y, 1}$ & 0.36121 & 0.01844 & 0.36079 \\
$\sigma_{n y}^{2}$ & 0.02001 & 0.00153 & 0.01996 \\
$p_{n y, 11}$ & 0.98322 & 0.00744 & 0.98456 \\
$p_{n y, 00}$ & 0.93251 & 0.02667 & 0.93539 \\
$\mu_{t x, 0}$ & -0.20619 & 0.02203 & -0.20614 \\
$\mu_{t x, 1}$ & 0.56382 & 0.02258 & 0.56399 \\
$\sigma_{t x}^{2}$ & 0.02605 & 0.00187 & 0.02593 \\
$p_{t x, 11}$ & 0.98503 & 0.00642 & 0.98598 \\
$p_{t x, 00}$ & 0.93265 & 0.02687 & 0.93487 \\
$\sigma_{n y, t x}$ & 0.00819 & 0.00156 & 0.00820 \\
$p_{11}$ & 0.98113 & 0.00775 & 0.98240 \\
$p_{00}$ & 0.93069 & 0.02523 & 0.93472 \\
$p_{V, 11}$ & 0.96516 & 0.03974 & 0.97721 \\
$p_{V, 00}$ & 0.96206 & 0.02731 & 0.96879 \\
\hline
\end{tabular}

Note: The selected example presents the case of two states with high and similar shares of U.S. GDP, New York with $7.68 \%$ and Texas with $7.95 \%$.

Table 5: Dynamic synchronization estimates between California and Vermont

\begin{tabular}{cccc}
\hline & Mean & Std. Dev. & Median \\
\hline$\mu_{n y, 0}$ & -0.05433 & 0.01651 & -0.05441 \\
$\mu_{n y, 1}$ & 0.38183 & 0.01736 & 0.38236 \\
$\sigma_{n y}^{2}$ & 0.02320 & 0.00180 & 0.02314 \\
$p_{n y, 11}$ & 0.97917 & 0.00855 & 0.98015 \\
$p_{n y, 00}$ & 0.94655 & 0.01969 & 0.94817 \\
$\mu_{t x, 0}$ & -0.12031 & 0.02875 & -0.11942 \\
$\mu_{t x, 1}$ & 0.44574 & 0.03106 & 0.44586 \\
$\sigma_{t x}^{2}$ & 0.05672 & 0.00447 & 0.05647 \\
$p_{t x, 11}$ & 0.97762 & 0.00882 & 0.97884 \\
$p_{t x, 00}$ & 0.94139 & 0.02105 & 0.94373 \\
$\sigma_{n y, t x}$ & 0.01574 & 0.00245 & 0.01561 \\
$p_{11}$ & 0.97829 & 0.00895 & 0.97956 \\
$p_{00}$ & 0.94627 & 0.02031 & 0.94897 \\
$p_{V, 11}$ & 0.97412 & 0.02714 & 0.98243 \\
$p_{V, 00}$ & 0.93864 & 0.03777 & 0.94518 \\
\hline
\end{tabular}

Note: The selected example presents the case of the states with the highest and the lowest shares of U.S. GDP, California with $13.34 \%$ and Vermont with $0.18 \%$. 
Table 6: Stationary synchronization between individual states and the entire United States

\begin{tabular}{llllll}
\hline State & Sync & State & Sync & State & Sync \\
\hline Alabama & 0.79 & Maine & 0.74 & Ohio & 0.84 \\
Arizona & 0.71 & Maryland & 0.52 & Oklahoma & 0.19 \\
Arkansas & 0.62 & Massachusetts & 0.56 & Oregon & 0.72 \\
California & 0.80 & Michigan & 0.85 & Pennsylvania & 0.79 \\
Colorado & 0.32 & Minnesota & 0.75 & Rhode Island & 0.65 \\
Connecticut & 0.79 & Mississippi & 0.75 & S. Carolina & 0.87 \\
Delaware & 0.77 & Missouri & 0.85 & S. Dakota & 0.54 \\
Florida & 0.70 & Montana & 0.34 & Tennessee & 0.84 \\
Georgia & 0.86 & Nebraska & 0.39 & Texas & 0.33 \\
Idaho & 0.57 & Nevada & 0.78 & Utah & 0.47 \\
Illinois & 0.75 & N. Hampshire & 0.44 & Vermont & 0.69 \\
Indiana & 0.83 & New Jersey & 0.77 & Virginia & 0.88 \\
Iowa & 0.59 & New Mexico & 0.49 & Washington & 0.69 \\
Kansas & 0.72 & New York & 0.72 & Wisconsin & 0.75 \\
Kentucky & 0.77 & N. Carolina & 0.91 & W. Virginia & 0.45 \\
Louisiana & 0.31 & N. Dakota & 0.24 & Wyoming & 0.25 \\
\hline
\end{tabular}

Note: The table reports the stationary synchronization for the period August 1979 to March 2013. These estimates correspond to the ergodic probability that the phases of the state business cycles and U.S. business cycles are the same, i.e., $\operatorname{Pr}\left(V_{t}=1\right)$. The index used to measure the national business cycle is the Chicago Fed National Activity Index (CFNAI). 
Figure 1: Simulation of changes in synchronization of cycles

$$
z=1
$$
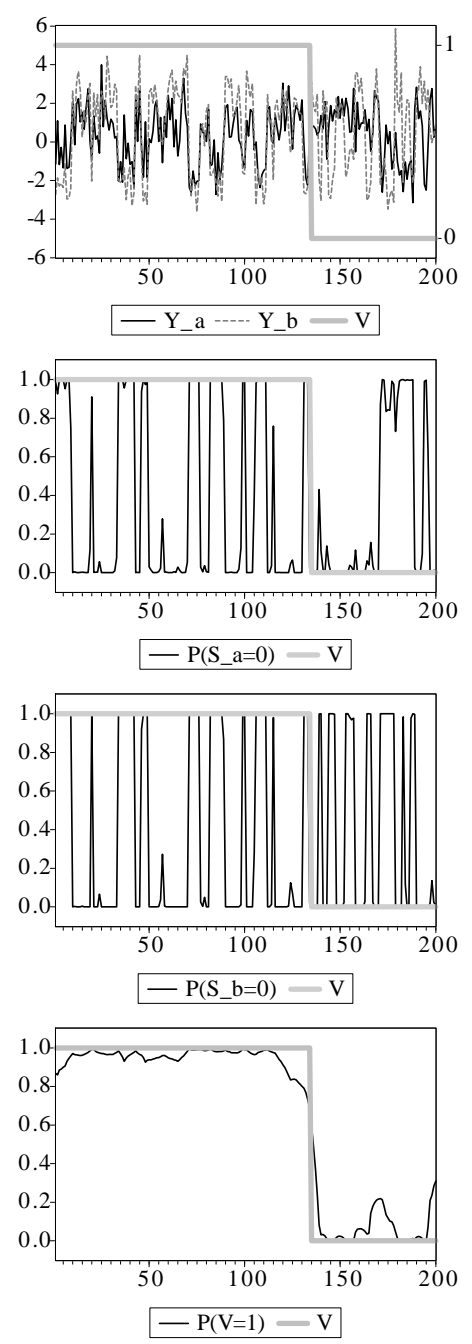

$z=2$
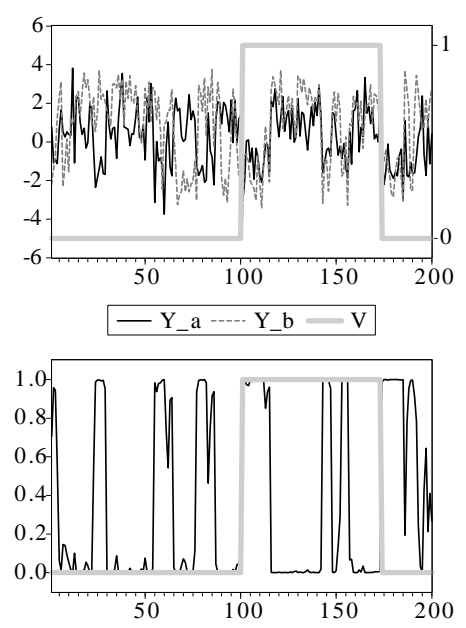

$-\mathrm{P}\left(\mathrm{S} \_\mathrm{a}=0\right)-\mathrm{V}$
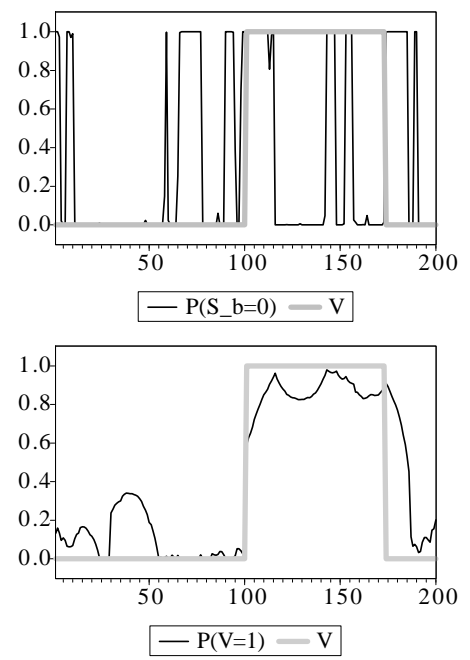

$z=3$
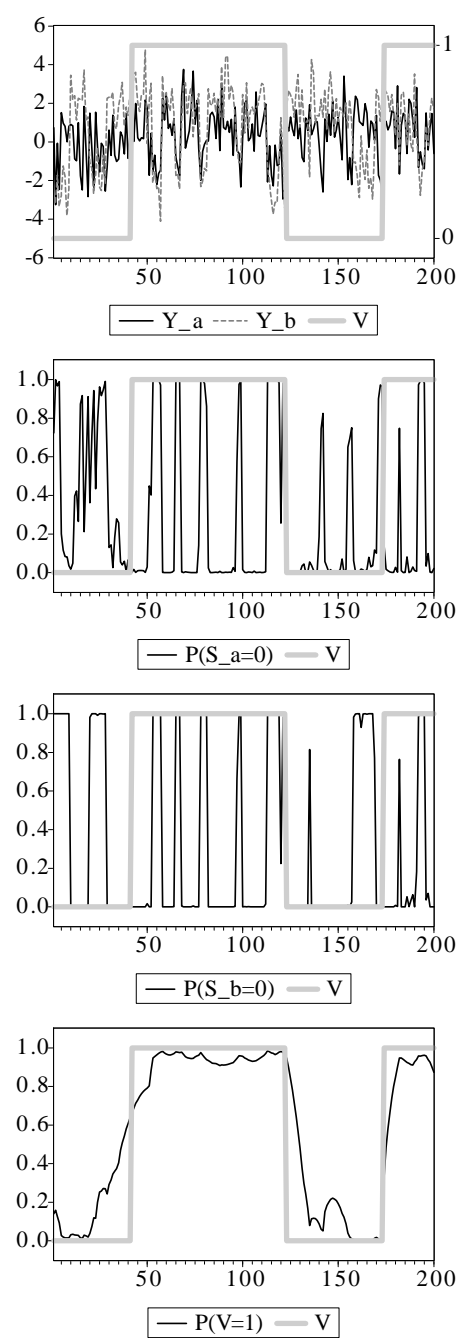

Note: The figure plots one simulation for the cases of 1, 2 and 3 changes in the synchronicity of cycles. For each case, the top panels plot the generated pair of time series along with the indicator variable of synchronization changes. The two middle panels plot the probabilities of a low mean regime associated with each time series, along with the indicator variable as reference. The bottom panels plot the estimated dynamics of the indicator variable along with the real one. 
Figure 2: Histograms of the performance of regime inferences
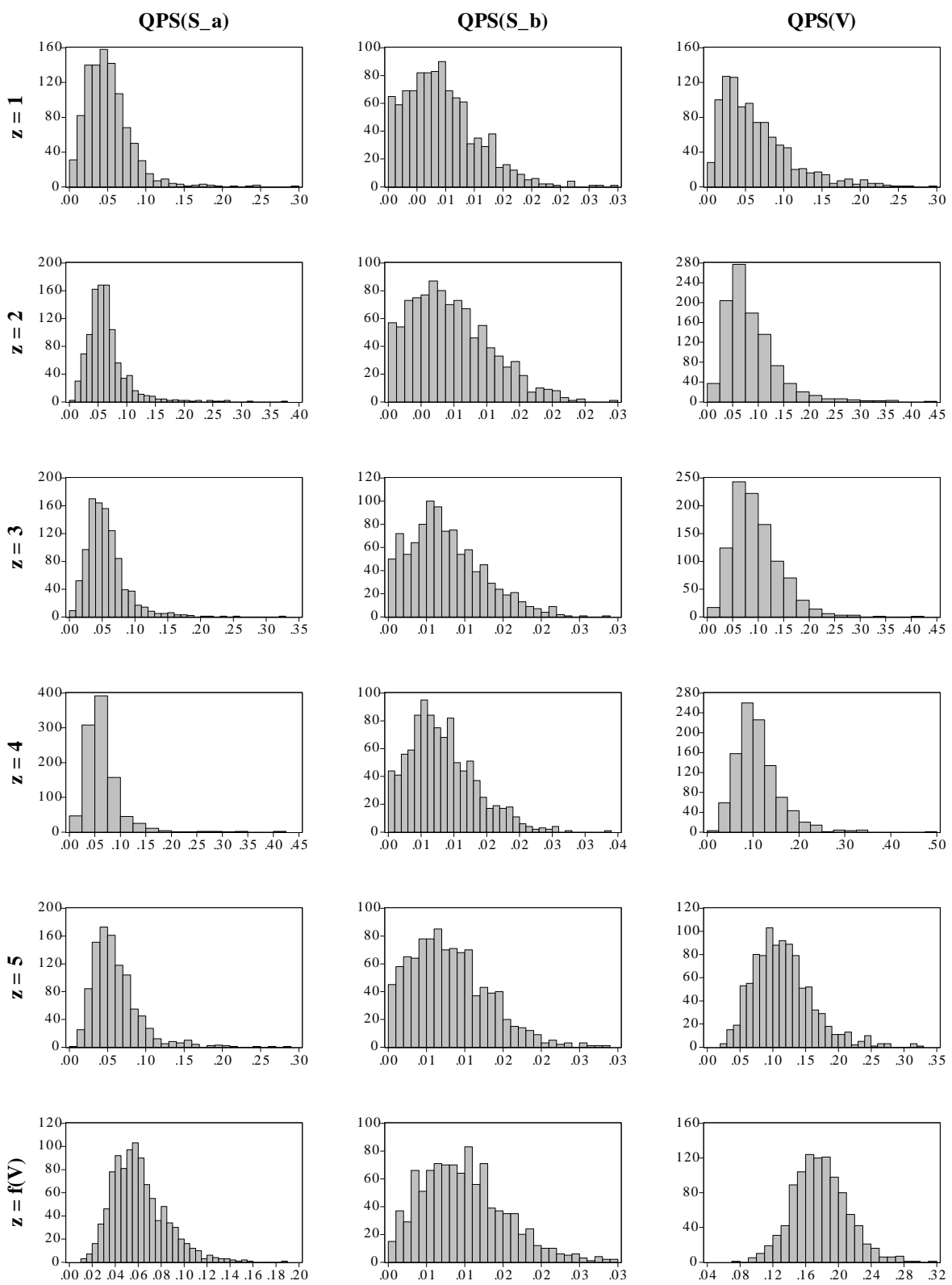

Note: The figure plots the histograms based on the 1,000 replications of the Quadratic Probability Score associated with the state variables for different numbers of synchronization changes, $z$. 
Figure 3: Dynamic synchronization between selected states

Chart A. New York and Texas

Recession probability for NY

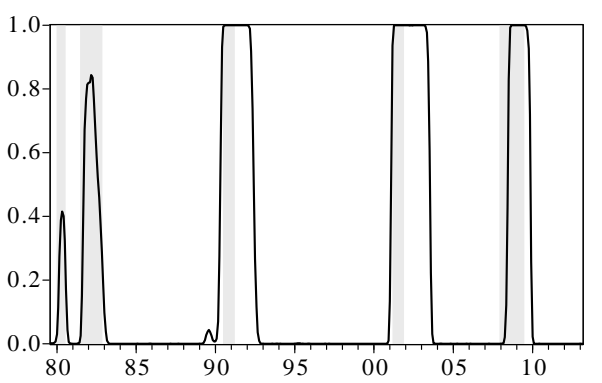

Recession probability for TX

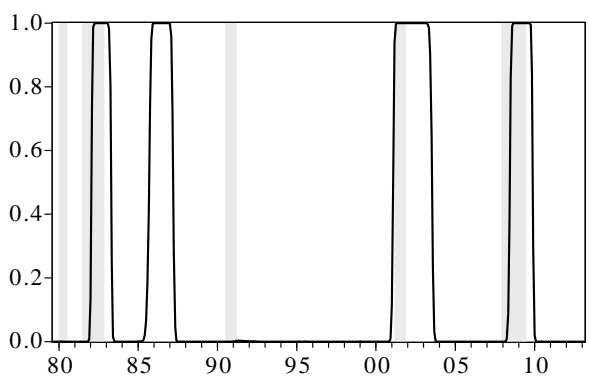

Dynamic Synchronization for NY and TX

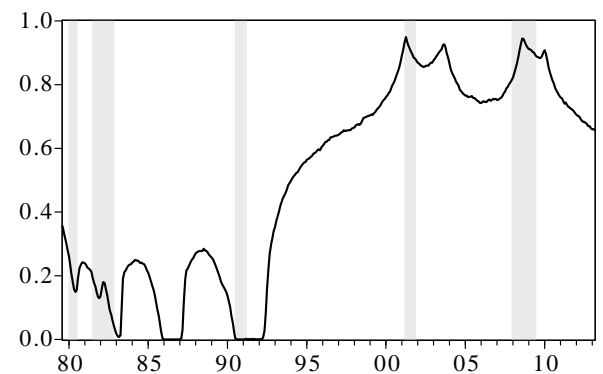

Chart B. California and Vermont

Recession probability for CA

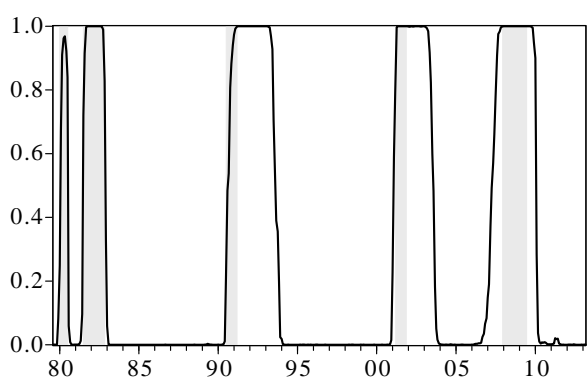

Recession probability for VT

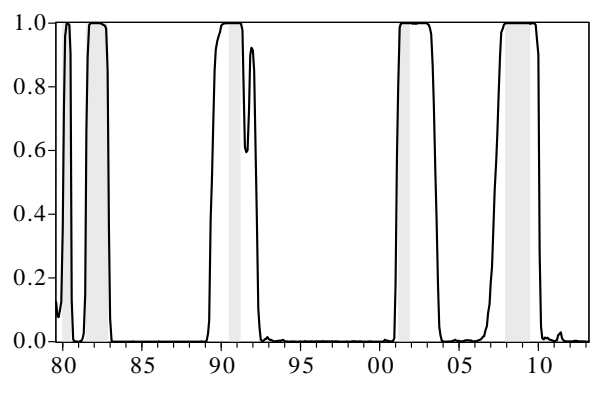

Dynamic Synchronization for CA and VT

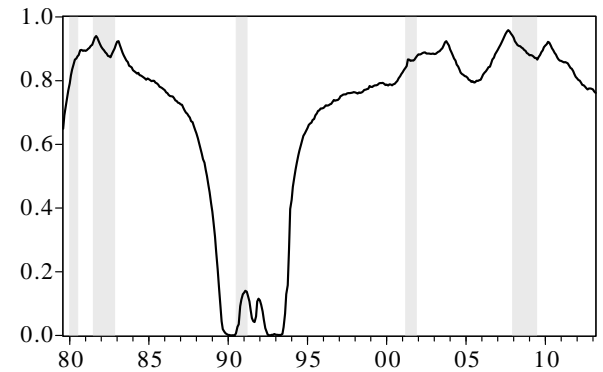

Note: The figure plots the output estimation for two selected pairwise models. Chart A plots the probability of recession for New York and Texas along with their dynamic synchronization. Chart B plots the probability of recession for California and Vermont along with their dynamic synchronization. Shaded areas correspond to NBER recessions. 
Figure 4: Dynamic synchronization maps of U.S. states across recessions
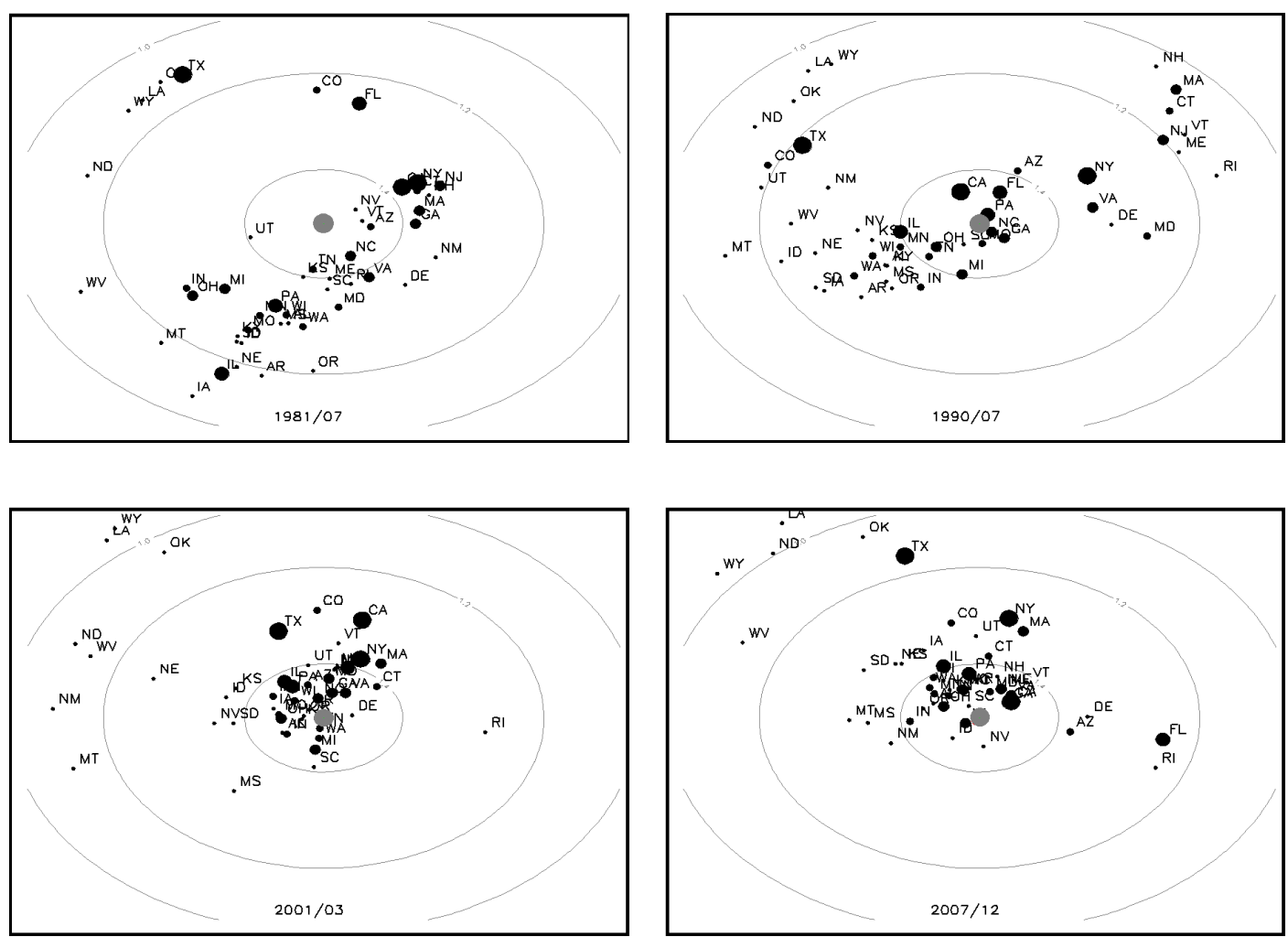

Note: Each chart in the figure plots the dynamic multi-dimensional scaling map based on the synchronization distance of the business cycle of U.S. states for different periods. The distances are normalized with respect to the U.S. national economic activity, the grey point in the centre. The size of the points refer to the GDP share of the corresponding state. If two states are placed in the same orbit, they are equally in sync with the United States. The full animated version of the synchronization mapping is available at https://sites.google.com/site/daniloleivaleon/media. 
Figure 5: Grouping pattern based on business cycle phases

Chart A. Ergodic synchronization map of U.S. states

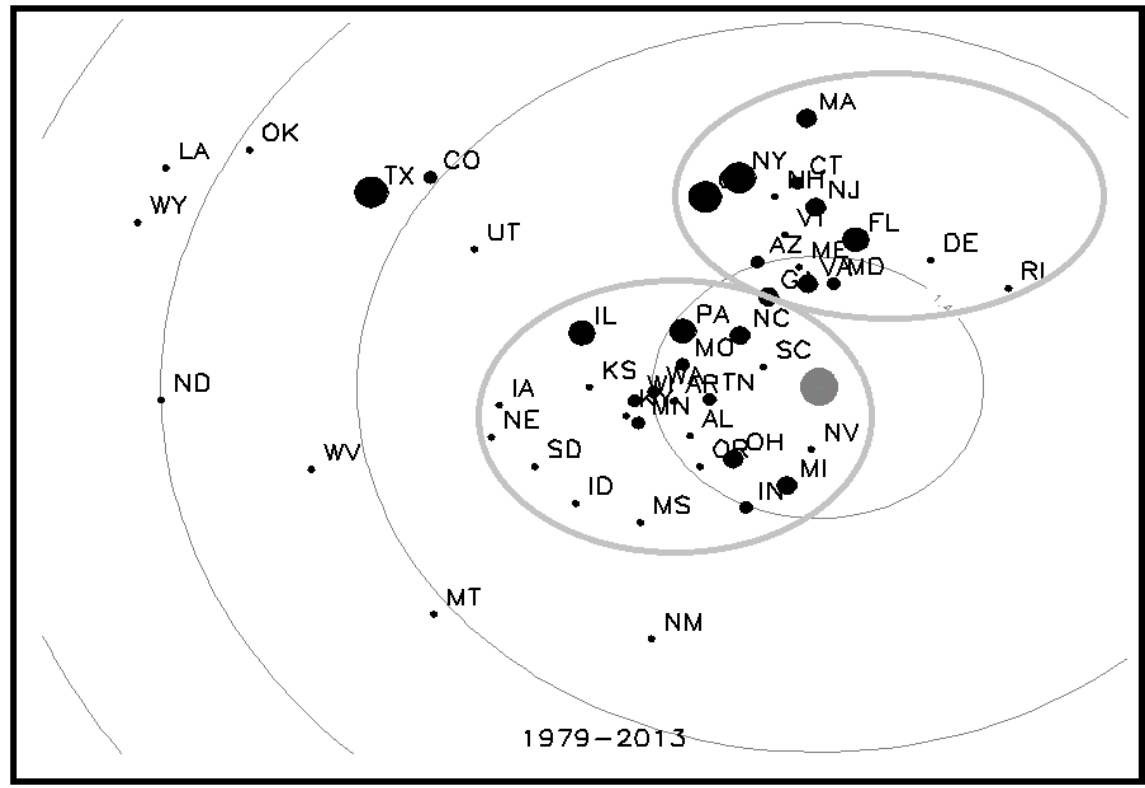

Chart B. Clustering pattern obtained in Hamilton and Owyang (2012)

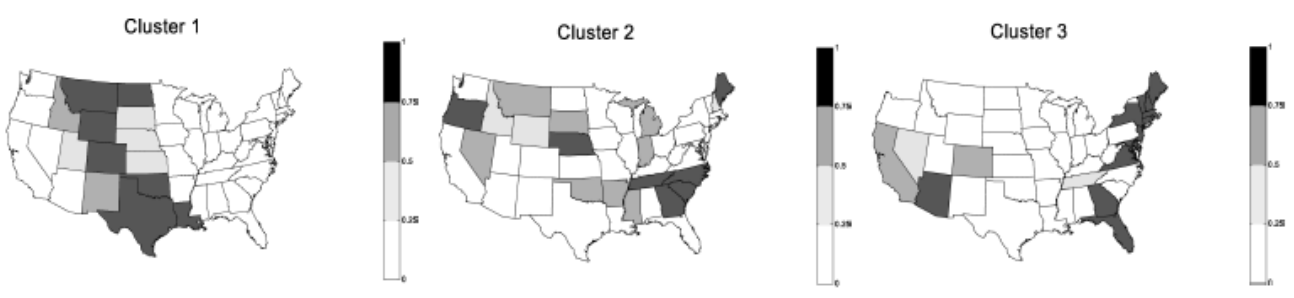

Note: Chart A plots the multi-dimensional scaling map based on the stationary synchronization distance of the business cycle characteristics of U.S. states for the sample August 1979 to March 2013. The distances are normalized with respect to the U.S. National Economic Activity, the grey point in the centre. If two states are placed in the same orbit, they are equally in sync with the United States. The ovals refer only to groups. The full animated version can be found at the author's web page. In Chart B, the shading for each state indicates the probability that the state belongs to any given cluster. Source: Hamilton and Owyang (2012) 
Figure 6: Synchronization between individual states and the entire U.S.

Chart A. Stationary synchronization
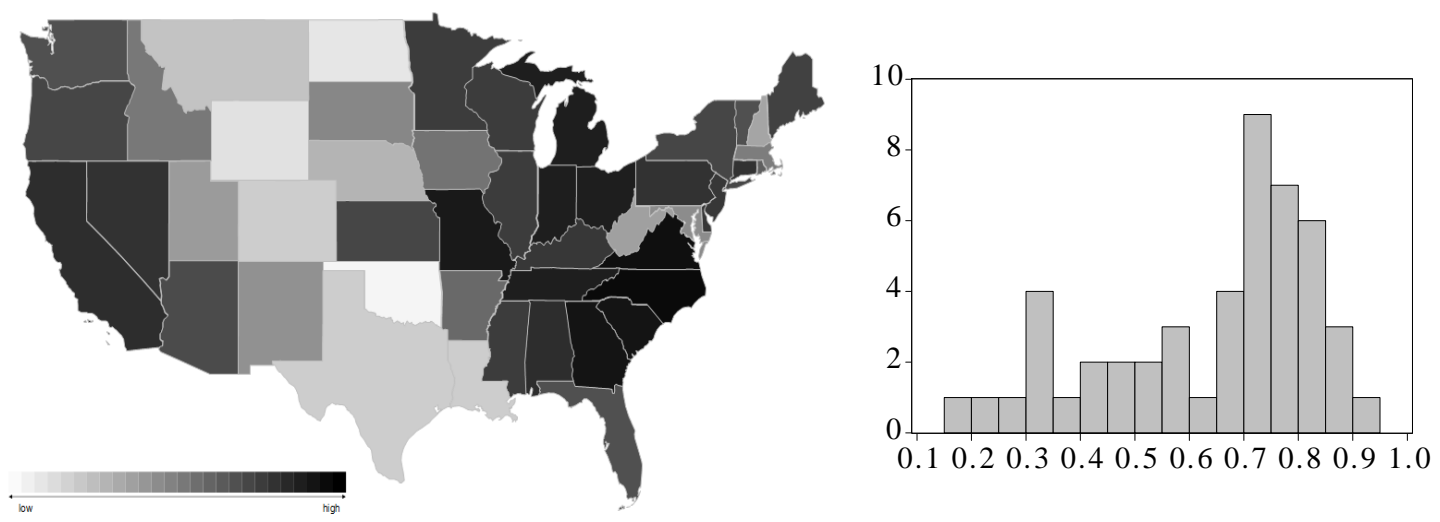

Chart B. Concordance based on Owyang et al. (2005)
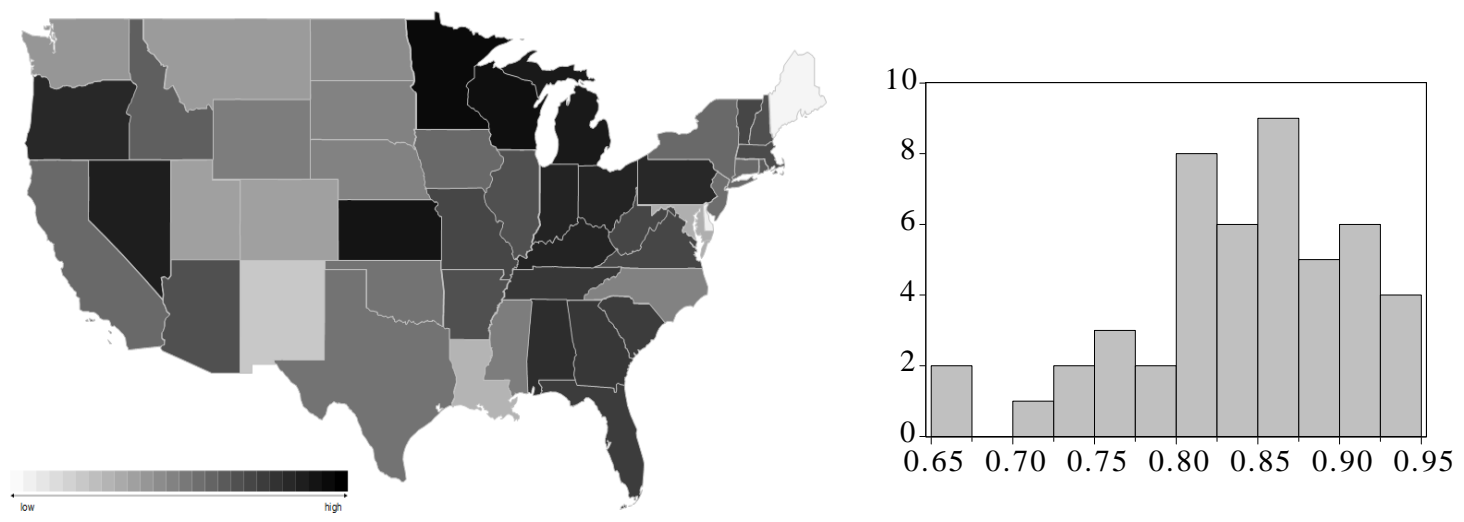

Note: Chart A of the figure plots a thematic map of the United States based on the stationary synchronization between each state and the national business cycle along with the histogram corresponding to data in Table 6. A darker state represents a higher synchronization. Chart B plots a thematic map of the United States using the concordances obtained in Owyang et al. (2005) along with the corresponding histogram. 
Figure 7: Synchronization network of the U.S. states across recessions

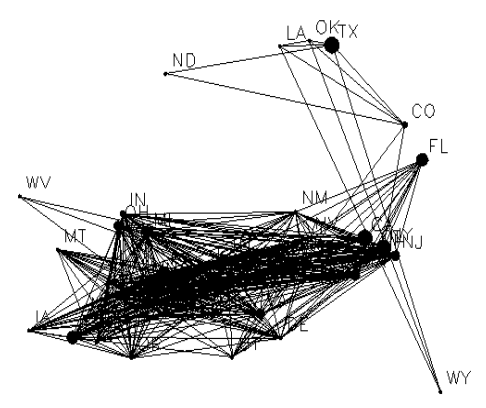

$.1981 / 07$

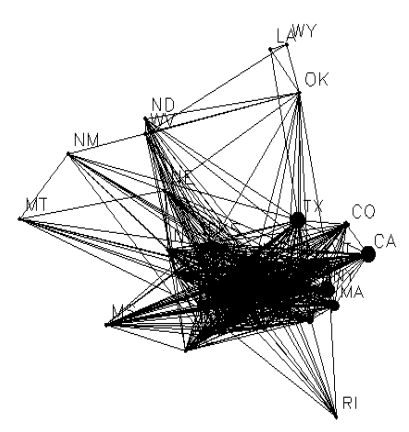

$.2001 / 03$

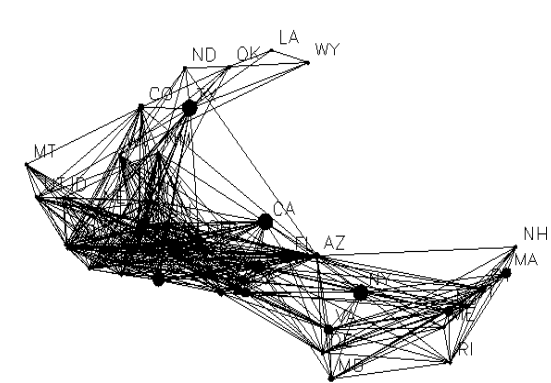

$.1990 / 07$

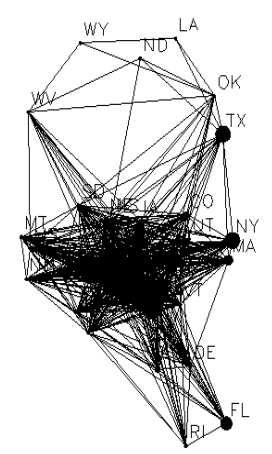

$.2007 / 12$

Note: The figure plots the interconnectedness in terms of synchronization between the business cycle phases of U.S. states. Each node represents a state and each line represents the link between two states, which takes place only if $\operatorname{Pr}(V t=1)>0.5$. The full animated version can be found at https://sites.google.com/site/daniloleivaleon/media. 
Figure 8: Dynamic closeness centrality of the U.S. synchronization network

Chart A. Smoothed prob.-based centrality

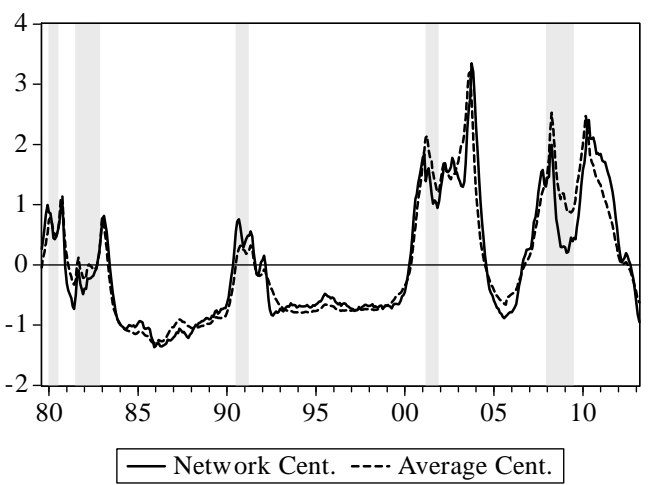

Chart B. Filtered prob.-based centrality

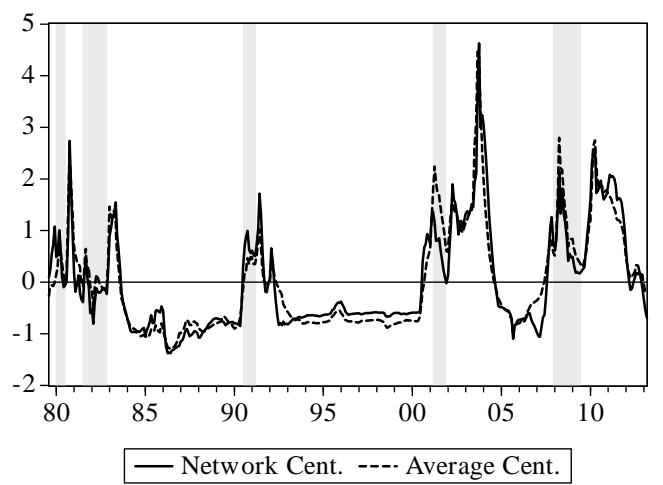

Note: Chart A of the figure plots the two measures of centrality of the Markov-switching synchronization network based on the smoothed probabilities, $\operatorname{Pr}\left(V_{t}=1 \mid \psi_{T}\right)$. The solid line plots the network closeness centrality defined in Equation (52) and the dotted line plots the average centrality, as defined in Equation (53). Chart B of the figure plots the same measures as in Chart A, but based on the filtered probabilities, $\operatorname{Pr}\left(V_{t}=1 \mid \psi_{t}\right)$. All the series in the figure are standardized to facilitate their comparison. Shaded bars refer to the NBER recessions.

Figure 9: Dynamic clustering coefficient of the U.S. synchronization network

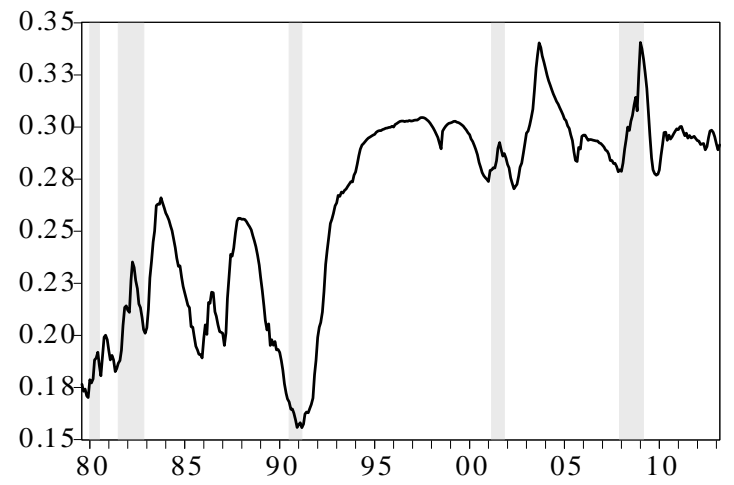

Note: The figure plots the time-varying clustering coefficient of the Markov-Switching Synchronization Network for U.S. states. Shaded bars refer to the NBER recessions. 